\title{
MODEL-BASED TRACKING OF MOVING OBJECTS IN CLUTTERED ENVIRONMENTS
}

\author{
BY \\ BASILIS GIDAS (Division of Applied Mathematics, Brown University, Providence, RI 02912), \\ FERNANDO CARVALHO GOMES (Departamento de Ciência da Computação, Universidade \\ Federal do Ceará, Fortaleza, CE 60160-120, Brazil), \\ AND \\ CHRISTOPHER ROBERTSON (Agency.com, 55 William Street, Suite 700, Wellesley, MA \\ $02481)$
}

\begin{abstract}
We explore a coherent statistical/Bayesian framework for tracking rigid or non-rigid objects in highly cluttered environments. The procedure involves three basic models: (i) an object representation, (ii) a dynamic model, and (iii) a data or observation model. We employ two object representations - deformable templates, and a hierarchical/syntactic model; the deformations of the template are parametrized by a finite number of global parameters viewed as generalized coordinates of the model; they determine the state space of the tracking problem. The dynamic model describes the evolution of the generalized coordinates (or the corresponding state vector); our dynamic model is a non-Gaussian Markov chain whose transition probabilities are derived from Lagrangian mechanics. We introduce two data models: one nonlinear and one linear (Gaussian); the design of the two models uses different image processing techniques. The nonlinear filtering problem induced by three models is solved both by the Extended Kalman Filter (EKF), and an iterative algorithm - to be referred to as the Monte Carlo Filter (MCF) introduced in the statistic literature and first employed in Computer Vision by Blake and Isard. We present several tracking experiments with real video sequences. In each experiment we implement both MCF and EKF, and their performances are compared. Overall, we find that $\mathrm{MCF}$ is more robust than EKF in situations with considerable clutter and/or occlusions, but EKF is comparable and sometimes more accurate than MCF in situations with less occlusion and degradation. Our studies also demonstrate that when the motion
\end{abstract}

Received August 8, 2001.

2000 Mathematics Subject Classification. Primary 68T45, 62H35, 65C60.

The first author was partially supported by ARO/MURI grant DAAH04-96-1-0445, NSF Grant DMS0074276, and CNPq Grant 301179/00-0.

The second author was supported in part by CNPq Grant 300708/93-9.

E-mail address: gidas@dam.brown.edu

E-mail address: carvalho@lia.ufc.br

E-mail address: crobertson@agency.com 
has sharp discontinuities in direction, nonlinearities in the dynamic model are key for successful tracking; put differently, the deficiencies of linear dynamics models cannot be easily compensated with more sophisticated data models.

1. Introduction. Tracking moving objects on the basis of video image sequences, and more generally, analysing visual motion, is a central task in computer vision. Its applications include traffic monitoring (e.g., monitoring vehicles on highways or aircraft in airport scenes), surveillance (e.g., tracking people in subways or banks), dynamic medical imaging (e.g., analysing the motion of a beating heart on the basis of ultrasound image sequences), autonomous or mobile robots, animation, and video image compression.

In this paper we explore a coherent statistical/Bayesian framework for tracking rigid or non-rigid objects moving in highly cluttered and degraded environments. The framework involves three basic models: (i) an object representation, i.e., a model that articulates the overall shape architecture of an object in a library of objects, together with the shape's random deformation (position, orientation, and non-rigid elastic type deformations); (ii) a dynamic model that describes a prior distribution on an object's plausible dynamical motions; and (iii) a data or observation model that relates the image gray-level data, at each (video) frame, to the object and dynamic models, and articulates the random variability of the image data due to the various factors of uncertainty such as clutter occlusion, noise, blur, and other degradation mechanisms.

The combination of the three models leads to a nonlinear filtering problem which is equivalent to a Hidden Markov Model (HMM). The HMM representation is convenient for the design of dynamic and data models. The solution of the nonlinear filtering problem requires a computational (or filtering) algorithm which may be viewed as the fourth component of the tracking problem. The design of the object, dynamic, and data models are driven by the objective of making the computations feasible and achieving tracking in real-time. We introduce two different data models, and explore two different types of computational algorithms: the Extended Kalman Filter (EKF), and an iterative algorithm - to be referred to as the Monte Carlo Filter (MCF)-introduced in the statistics literature [13] and first employed in Computer Vision by Blake and Isard [16, 3]. Experimental comparisons of the two algorithms are briefly discussed below, and in more detail in $\S 6$.

Our object, dynamic, and data models are described in Sections 2, 3, and 4, respectively. In brief:

Object Models: We explore two types of object representation-deformable templates $[14,4]$, and a hierarchical/syntactic model [20]; the latter is closely related to ContextFree-Grammars. In the deformable template representation, the transformations of the template are parametrized in terms of location $(\alpha)$, orientation $(\theta)$, and a deformation vector field $u(\xi)$ where $\xi$ represents a point on the object in the body frame. The deformation field $u(\xi)$ is parametrized (see $\S 2.1$ ) by $N_{d}$ global parameters $\lambda \in R^{N_{d}}$. From the point of view of the dynamic model (and to some extent of the data model), the main ingredient of the object representation is the parameter vector $q=(\alpha, \theta, \lambda)^{\top}$ to be referred to as the generalized coordinate vector-or its associated state vector 
$X(t)=(q(t), \dot{q}(t))^{\top}, t \geq 0$ (throughout this paper $\top$ will denote transposition of vectors or matrices).

In the case the object and its deformations are star-shaped, we explore an additional representation [20] described by a context-free-grammar. This representation is used only in devicing one of our data models; we do not know how to design a dynamic model based on this representation; however this should be possible, and due to its hierarchical/ syntactic structure, the representation should allow dynamic programming computations both at a frame and across frames.

Dynamic Model: The dynamic model describes the evolution $q(t)$ of the generalized coordinates $q=(\alpha, \theta, \lambda)^{\top}$ - equivalently, the evolution of the state vector $X(t)=$ $(q(t), \dot{q}(t))^{\top}$, or its (time) discretization $X_{k}=X(k \delta t), k=0,1,2, \ldots$ The dynamic model may be viewed as a prior distribution on the set of plausible trajectories of an object. It is not required to represent the precise motion of an object, but only to articulate generic properties of it. For example, it should describe trajectories that are smooth most of the time, but it should also allow abrupt changes in the direction of motion (e.g., a vehicle or an aircraft may take sharp turns). A dynamic model may be represented by a Markov chain (not necessarily of first order) or, more generally, by a stochastic process. Gaussian models such as the autoregressive (AR) or autoregressive/moving average (ARMA) do not allow discontinuities in the direction of motion, and thus, we believe, are not adequate for real-world tracking tasks (see, however, $[16,3]$ ). Our dynamic models are non-Gaussian Markov chains. The design of suitable non-Gaussian chains, i.e., the design of their transition probabilities, is not a straightforward matter. The issue is analogous to the design of Markov Random Fields (MRF) as priors in imaging processing tasks; it is the Gibbs representation [11] of MRF (via the Hammesley-Clifford theorem) that provides a powerful device for designing appropriate ("physically meaningful") MRF.

This device could also be used in the design of Markov chain dynamical models, but a different rationale (than the one used in the design of MRF for static images) is needed for choosing the potential functions. We believe that the natural framework for this is that of classical mechanics - in our setup, that of Lagrangian mechanics $[22,24]$. In this framework, the Gibbs potentials are derived from more fundamental quantities that depend on the physical (and geometric) properties of the moving objects and of the environment in which the objects move, as well as on the external forces that may act on the object. Our non-Gaussian Markov chains are derived from Lagrangian mechanics by incorporating three types of energies (kinetic, dissipation, and strain), and suitable random external forces. In Section 3, we spell out the resulting Gibbs potentials, their nonlinearities, and their parametrizations in terms of physical or geometric parameters. Lagrangian mechanics for related image analysis problems was first used in [26].

Data Models: Given a sequence of observations $\left\{Y_{k}: k \geq 0\right\}$, a data or observation model is defined by specifying the conditional probability (likelihood function) $P\left(Y_{k} \mid X_{k}\right)$ at each frame $k \geq 0$. We employ two different data models - one linear (i.e. Gaussian) and one nonlinear; in either case, the filtering problem is nonlinear due to the nonlinearity of our dynamic model. The filtering problem with the linear data model is solved using the EKF, while the one with the nonlinear data model uses the MCF. In the nonlinear 
model, $Y_{k}$ represents the raw gray-level data at frame $k$ together with differences between the gray-levels at frame $k$ and a previous frame (we use $k-2$; in general, the choice of the previous frame depends on the time resolution). The basic idea for designing the nonlinear data model is this: a given $X_{k}$ determines a plausible instantiation (location and pose) of the object (in the implementation $X_{k}$ represents one of the predictive samples generated by the MCF (see $\S 5)$ ). Comparing the data inside the object with the data outside the object, we design a contrast statistics (or cost function) $D\left(Y_{k}, X_{k}\right)$; then $D$ is used to define $P\left(Y_{k} \mid X_{k}\right.$ ) (see $\left.\$ 4.1\right)$. We have explored both classical and nonparametric (rank type statistics) in defining $D$. But any reasonable choice of $D$ makes the tracking algorithm computationally heavy if not impossible. In order to bypass this difficulty we explore the structure of the Monte Carlo Filter algorithm, and "approximate" our basic data model by a new quantity (see $§ 5$ ) that makes computations feasible (in fact, tracking is attained in real-time).

In the linear data model, $Y_{k}$ represents an estimate of the generalized coordinates at frame $k$. We assume that up to an additive Gaussian noise, the estimate $Y_{k}$ is linearly related to the true generalized coordinates. The estimate $Y_{k}$ is obtained by a segmentation algorithm which uses the gray-levels at the current frame and the output of the predictive step of EKF (see $§ 4.2$ for details).

In Section 6, we present several tracking experiments with real images sequences. In each experiment, we implement both the EKF and the MCF, and their performances are compared. Overall we found that the MCF is more robust than the EKF in situations with considerable clutter and/or occlusion, but in other situations the EKF is comparable and sometimes slightly more accurate than the MCF. Our studies demonstrate that when the motion has sharp discontinuities in direction, nonlinearities in the dynamic model are key for good tracking quality; put differently, in these cases, the deficiencies of linear dynamic models cannot be easily compensated with more sophisticated data models.

Similar, but different in details, approaches to tracking or tracking related problems have been promoted by several people $[16,3,2,5,19,27,28,29,15,18]$. Closer in spirit to our approach is that of Blake and Isard (see [3] and references cited therein). Their object representation, and dynamic and data models differ from those of ours in a number of ways. B-splines form the basis of their object representation, and the design of their data model is based on different types of image processing techniques than the ones used here. While our data model is considerably different than theirs, perhaps the main difference lies in the dynamic model; they employ linear autoregressive type models, while our dynamic models are nonlinear and are derived from the basic principles of Lagrangian mechanics. As we mentioned above, we believe that nonlinear dynamics are key in some problems; in a sense, it seems that nonlinearity is more dispensable in the data models than in the dynamic models.

The organization of the paper is as follows. Section 2 describes our object representations (deformable templates in $§ 2.1$ and hierarchical/syntactic model in $§ 2.2$ ). Section 3 contains our dynamical model; some technical parts of this section are described in the Appendix. Data models are described in Section 4 ( $\$ 4.1$ contains the nonlinear data model, and $\S 4.2$ the linear data model). Section 5 presents the Monte Carlo Filter algorithm and a modification of our nonlinear data model that makes computations feasible. 
Finally, Section 6 contains our experimental results and comparisons of the EKF and MCF performances.

2. Object Representations. In this section we present two object representations - deformable templates and a hierarchical/syntactic model. We address representations for 2-D deformable objects, as we are interested in tracking the images of the objects on the image plane, and not their trajectories in the 3-D world; thus, throughout this paper, the term "object" will refer to the image of an object. We note that the image of an object is a deformable object even if the original object is rigid.

2.1. Deformable Templates. The deformable template methodology [14, 4] represents objects or patterns in terms of templates and a set of transformations acting on the template. The template of a specific object will be denoted by $V$; we view $V$ as a 2-D region (not as the boundary of the object). The transformations of the template are expressed by a translation $\alpha \in \mathbb{R}^{2}$, and a vector field $v(\xi) \in \mathbb{R}^{2}$ with $\xi \in V$ representing a point on the object in the body frame. The corresponding point $x$ in the inertial frame is given by $x=\alpha+v(\xi)$. The vector field $v(\xi)$ may be written as $v(\xi)=R(\theta) w(\xi)$, where $\theta$ is an angle of rotation of the body frame relative to the inertial frame, $R(\theta)$ is the standard rotation matrix

$$
R(\theta)=\left(\begin{array}{rr}
\cos \theta & -\sin \theta \\
\sin \theta & \cos \theta
\end{array}\right),
$$

and $w(\xi)$ denotes the new (transformed) position of $\xi$ in the body frame. Writing $w(\xi)=$ $\xi+u(\xi), u(\xi)$ is called the deformation vector field and describes elastic deformations (including scales). Thus after translation, rotation, and deformation, a point $\xi \in V$ in the body frame is transformed into a point $x$ in the inertial frame given by

$$
\begin{aligned}
x & =\alpha+v(\xi)=\alpha+R(\theta) w(\xi) \\
& =\alpha+R(\theta) \xi+R(\theta) u(\xi) .
\end{aligned}
$$

There are various ways of parametrizing $u(\xi)$ (e.g., via superquadrics [26]). Here we begin with a parametrization of $v(\xi)$, and then derive a parametrization of $u(\xi)$. The vector $v(\xi)$ is approximated by expanding it in a basis, and then truncating the series expansion. It is more convenient to expand $v$ in an unormalized (rather than orthonormal) basis. More precisely, let $\xi=\left(\xi_{1}, \xi_{2}\right)^{\top}$ and

$$
e_{m}(\xi)=\left(\xi_{1}^{m}, \xi_{1}^{m-1} \xi_{2}, \xi_{1}^{m-2} \xi_{2}^{2}, \ldots, \xi_{2}^{m}\right)^{\top}, m=1,2, \ldots
$$

Then we approximate $v(\xi)$ by

$$
v(\xi)=A_{1} \xi+A_{2} e_{2}(\xi)+\ldots+A_{n} e_{n}(\xi)
$$


where $A_{m}$ is a $2 \times(m+1)$ matrix $(m=1,2, \ldots, n)$. We assume that the determinant of $A_{1}$ is different from zero, so that the transformation (2.2a) is locally invertible. Then $A_{1}$ may be written in terms of more meaningful parameters, i.e., it may be written as

$$
A_{1}=R(\theta)\left(I+\Lambda_{1}\right)
$$

where $R(\theta)$ is the rotation matrix $(2.1)$, I is the $2 \times 2$ identity matrix, and $\Lambda_{1}$ is a symmetric matrix that can be written as

$$
\Lambda_{1}=\left(\begin{array}{ll}
\lambda_{1} & \lambda_{3} \\
\lambda_{3} & \lambda_{2}
\end{array}\right) .
$$

The parameters $\lambda_{1}, \lambda_{2}$, and $\lambda_{3}$ represent scales along horizontal, vertical, and diagonal directions, respectively. Using $R(\theta)$ we may write $A_{m}$ as

$$
A_{m}=R(\theta) \Lambda_{m}, m=2, \ldots, n
$$

where the $\Lambda_{m}$ 's are $2 \times(m+1)$ matrices with at most $2(m+1)$ independent parameters. Let $\lambda^{(1)}=\left(\lambda_{1}, \lambda_{2}, \lambda_{3}\right)^{\top}$, and collect the parameters of $\Lambda_{m}$ into a vector $\lambda^{(m)}, m=$ $2, \ldots, n$. Then the induced representation of the deformation vector field $u(\xi)$ is given by

$$
\begin{aligned}
u(\xi) & =\Lambda_{1} \xi+\Lambda_{2} e_{2}(\xi)+\ldots+\Lambda_{n} e_{n}(\xi) \\
& =S(\xi) \lambda
\end{aligned}
$$

where $\lambda=\left(\lambda^{(1)}, \lambda^{(2)}, \ldots, \lambda^{(n)}\right)^{\top}$, and $S(\xi)$ (defined by (2.5a)) is known as a "shape" matrix $; S(\xi)$ is a $2 \times N_{\lambda}$ matrix where $N_{\lambda}$ is the dimension of the vector $\lambda$ (note that $N_{\lambda}$ is at most $\left.n^{2}+3 n-1\right)$.

Our deformable template representation consists of (2.2) and (2.5) applied to every point $\xi$ on the template $V$. The vector $q=(\alpha, \theta, \lambda)^{\top}$ will be referred to as the generalized coordinate vector; its dimension is $3+N_{\lambda}$ (two for translation, one for rotation, and $N_{\lambda}$ for deformation parameters $\lambda$ ). The case when $\Lambda_{2}=\Lambda_{3}=\ldots=\Lambda_{n}=0$, corresponds to affine transformations.

The above object representation involves two choices: the template $V$ and the approximation (2.5) of the deformation field $u(\xi)$. The template $V$ is typically chosen to be a "canonical" instantiation of the object, but sometimes other shapes may also be chosen as templates. The approximation $(2.5)$ of $u(\xi)$ (i.e., the precise parametrization of the matrices $\Lambda_{m}, m=1, \ldots, n$, and even the degree $n$ of the approximation) could in principle be inferred from multiple images of the object. In this paper we choose it in an ad hoc manner (see $\S 6$ ). The choice of the approximation is driven by two competing factors: tracking algorithm computations that favor small dimensionality of $\lambda$ and accuracy of object representation that typically requires high dimensional $\lambda$. Our experiments $(\S 6)$ demonstrate that even simple approximations (such as the affine approximation) often give satisfactory results. 
2.2. Hierarchical/Syntactic Representation of Star-Shaped Objects. The representation described below works only for star-shaped objects (we require that not only the template, but also all its deformations during the motion, are star-shaped). The representation is a modification of that of [20] for convex sets; the modification for star-shaped objects results in a dramatic reduction in computation cost relative to the one for convex objects.

We denote the 2-D region of a star-shaped object by $O$ (thus $O$ could be the template $V$ or any of its deformations). Let $x_{o}$ be a point in $O$ relative to which $O$ is star-shaped. We fix a coordinate system with origin $x_{0}$. Then the boundary $\partial O$ of $O$ may be described by a radius function $r(\theta), 0 \leq \theta \leq 2 \pi$, with the properties $r(\theta)>0, r(0)=r(2 \pi)$, and $r(\theta)$ is single-valued. We approximate $\partial O$ by a sequence of polygons (equivalently, $O$ is approximated by a sequence of wedged-regions) as follows: let

$$
\mathcal{L}_{n}=\left\{\frac{2 \pi k}{2^{n}}: k=0,1, \ldots, 2^{n}-1\right\}
$$

be a dyadic division of $[0,2 \pi]$ at level $n=0,1,2, \ldots$ Note that

$$
\mathcal{L}_{n}=\mathcal{L}_{n-1} \bigcup\left\{\frac{2 \pi(2 k-1)}{2^{n}} \quad k=0,1, \ldots, 2^{n}\right\}
$$

i.e., an interval at level $n-1$ may be viewed as a concatenation (or union) of two adjacent intervals at level $n$. Set $r_{n, k}=r\left(\frac{2 \pi k}{2^{n}}\right)$, and let

$$
Z_{n, k}=\left(r_{n, k}, r_{n, k+1}\right), \quad k=0,1,2, \ldots, 2^{n}-1 .
$$

Then $Z_{n}=\left\{Z_{n, k}: k=0,1, \ldots, 2^{n}-1\right\}$ may be thought as representing a polygonal approximation (with $2^{n}$ vertices) of $\partial O$. We shall refer to $Z_{n, k}, k=0,1, \ldots, 2^{n}-1$, as the states of the approximation at level $n$. By (2.6), a state $Z_{n-1, k}$ at level $n-1$, may be thought as being obtained by "composition" of two states, $Z_{n, 2 k}$ and $Z_{n, 2 k+1}$, at level $n$; equivalently, the states at level $n$ may be thought as being generated from the states at level $n-1$ by the "production rule"

$$
Z_{n-1, k} \longmapsto Z_{n, 2 k} \circ Z_{n, 2 k+1}, \quad k=0,1, \ldots, 2^{n-1}-1 .
$$

The collection of states $\left\{Z_{n, k}: k=0,1, \ldots, 2^{n}-1, n=0,1, \ldots\right\}$ together with the production rules (2.7) is our hierarchical/syntactic representation of 2-D star-shaped objects. The representation may be described by a binary tree (see Fig. 2.1) whose nodes are indexed by $Z_{n, k}$ and whose edges represent the production rules (2.7). In practice, the tree is terminated at some level $N$, corresponding to an approximation of $O$ by a polygon with $2^{N}$ vertices. Moreover, if we quantize the range of $r(\theta)$ to a finite number of values, then the resulting representation is equivalent to a context-free-grammar [20, 21].

The above hierarchical/syntactic representation could, in principle, be combined with deformations similar to those in $\S 2.1$ subject to the constraint that the deformations preserve the star-shaped property. The resulting deformable hierarchical/syntactic models could be the basis for tracking. We do not pursue this direction in this paper. We use the representation only as the basis of a segmentation algorithm which, in turn, is used to devise one of our two data models (see $\S 4.2$ ). 


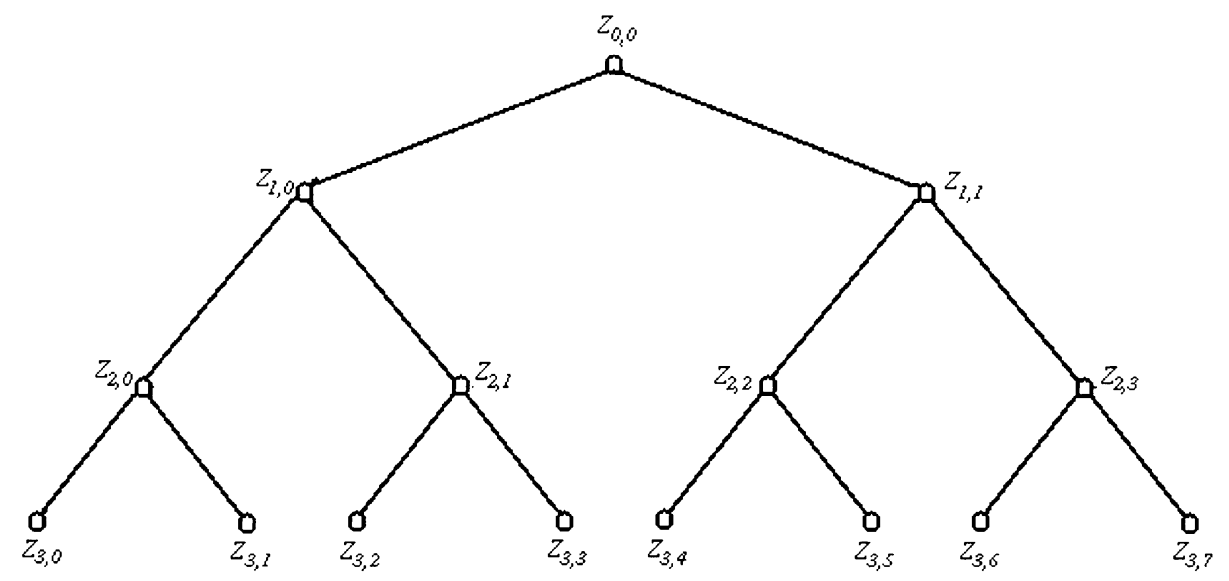

FIG. 2.1. Binary tree with $N=3$ levels

3. Dynamic Model. For continuous time, the dynamic model describes the evolution of the state vector $X(t)=(q(t), \dot{q}(t))^{\top}$ via a nonlinear stochastic differential equation. The discrete-time state vector $X_{k}=X(k \Delta t)$ forms a Markov chain with transition probabilities

$$
P\left(X_{k+1} \mid X_{k}\right)=p_{w}\left(M\left(X_{k}\right) X_{k+1}-\Psi\left(X_{k}\right)\right) \operatorname{det}\left(M\left(X_{k}\right)\right), k \geq 0
$$

and initial condition

$$
X_{0} \sim P\left(X_{0}\right) .
$$

Here $p_{w}(\cdot)$ is a probability density on $\mathbb{R}^{6+2 N_{\lambda}}$ (recall that $q(t)$ is $\left(3+N_{\lambda}\right)$-dimensional), and it corresponds to the distribution of an external random force. $M\left(X_{k}\right)$ is a $\left(6+2 N_{\lambda}\right) \times$ $\left(6+2 N_{\lambda}\right)$ matrix depending nonlinearly on $X_{k}$, and $\operatorname{det} M$ denotes its determinant. The vector $\Psi\left(X_{k}\right)$ depends also nonlinearly on $X_{k}$. At the end of this section we exhibit explicitly the nonlinearities and the parametrization of $M(\cdot)$ and $\Psi(\cdot)$. The choice of $p_{w}$ is also critical. This is discussed in $\S 6$ where we present our experiments.

As we mentioned in the Introduction, model (3.1a) is derived via the principles of Lagrangian mechanics using three types of energies - kinetic, dissipation, and strain. Below we describe these energies for continuous time, then derive the stochastic differential equation for $X(t)$. Our model (3.1) is derived by discretizing the equation for $X(t)$. The material below are more or less standard in Lagrangian mechanics; we present them in a way that exhibits the structure of our dynamic model - in particular, its nonlinearities and its parametrization.

3.1. Kinetic, Dissipation, and Strain Energies.

Kinetic Energy. The kinetic energy of the moving objects at time $t$ is defined by

$$
T=\frac{1}{2} \int_{V} \rho(\xi) \dot{x}^{\top} \dot{x} d \xi
$$


where $V$ is the template, $\rho(\xi)$ the mass density, and $\dot{x}$ the time derivative of the location $x$ in the inertial frame (see (2.2)). A simple calculation (see Appendix) gives

$$
T=\frac{1}{2} \dot{q}^{\top} M \dot{q}
$$

where $M=M(\theta, \lambda)$ is a symmetric matrix known as the mass matrix. Its matrix elements will be denoted by $M_{\alpha \alpha}, M_{\alpha, \theta}(\theta, \lambda), M_{\alpha, \lambda}(\theta), M_{\theta, \theta}(\lambda), M_{\theta, \lambda}(\lambda), M_{\lambda, \lambda}$. All of them are independent of $\alpha ; M_{\alpha, \alpha}$ and $M_{\lambda, \lambda}$ are also independent of $\theta$ and $\lambda ; M_{\alpha, \theta}$ is the only sub-matrix that depends on both $\theta$ and $\lambda$, while $M_{\alpha, \lambda}$ depends only on $\theta$, and $M_{\theta, \theta}$ and $M_{\alpha, \lambda}$ depend only on $\lambda$. The dependence on $\theta$ enters only through the rotation matrix (2.1). The formulas below show that only $M_{\theta, \theta}$ depends quadratically on $\lambda$; the other matrix elements are either independent of $\lambda$ or depend on $\lambda$ linearly. The explicit dependence on $R(\theta)$ and $\lambda$ is as follows (see Appendix):

$$
\left.\begin{array}{ll}
M_{\alpha, \alpha} & =m I \\
M_{\alpha, \theta}(\theta, \lambda) & =R(\theta) \sigma \mu+R(\theta) \sigma \Sigma \lambda \\
M_{\alpha, \lambda}(\theta) & =R(\theta) \Sigma \\
M_{\theta, \theta}(\lambda) & =\left(\mu_{11}+\mu_{22}\right)+2 \beta^{\top} \lambda+\lambda^{\top} \Gamma \lambda \\
M_{\theta, \lambda}(\lambda) & =\beta_{1}^{\top}+\lambda^{\top} \Gamma_{1} \\
M_{\lambda, \lambda} & =\Gamma
\end{array}\right\} .
$$

In these formulas, $\mathrm{I}$ is the $2 \times 2$ identity matrix, and $\sigma$ is the Pauli matrix

$$
\sigma=\left(\begin{array}{rr}
0 & -1 \\
1 & 0
\end{array}\right)
$$

The other parameters $\zeta=\left(m, \mu,\left\{\mu_{i, j}: i, j=1,2\right\}, \Sigma, \beta, \beta_{1}, \Gamma, \Gamma_{1}\right)$ are given (see Appendix) in terms of the moments up to the order $2 n$ of the template (here $n$ is the order of the approximation in (2.5)). These parameters are not all independent. In the case of affine transformations they all depend on the first five moments:

$$
\begin{aligned}
m & =\int_{V} \rho(\xi) d \xi \\
\mu & =\int_{V} \rho(\xi) \xi d \xi \\
\mu_{i, j} & =\int_{V} \rho(\xi) \xi_{i} \xi_{j} d \xi \quad, \quad i, j=1,2 .
\end{aligned}
$$

Moreover, if the body frame is centered at the center of mass, then $\mu=0$. If in addition there is a symmetry so that $\mu_{1,2}=0$, then $M_{\alpha, \theta}(\theta, \lambda)=0=M_{\alpha, \lambda}(\lambda)$, and the evolution $\alpha(t)$ of the translation coordinate $\alpha$ decouples (see below) from the evolutions of $\theta$ and $\lambda$. This remark is explored in our experiments.

In our implementations we choose the mass density $\rho(\xi)$ and then compute the parameters $\zeta$ analytically. In principle, one could parametrize the mass matrix elements with a sustainable set of identifiable parameters as in (3.4) and then estimated these parameters from data (more precisely, from the data in the filtering problem (see §5)). This is an interesting open problem not pursued in this paper. 
Dissipation Energy. In many applications damping is small, but its effect may be significant from the point of view of system stability. Hence the inclusion of damping is essential, and our experiments concur with this. We have explored the simplest type of damping forces that are proportional to the velocity field. In this case the "generalized" damping forces are derived [22] from Rayleigh's dissipation energy function

$$
D=\frac{1}{2} \int_{V} \gamma(\xi) \dot{x}^{\top} x d \xi,
$$

where $\gamma(\xi)$ is the dissipation density. The form of D is similar to that of the kinetic energy function (3.2) (simply replace $\rho(\xi)$ by $\gamma(\xi)$ ). Hence it can be written as

$$
D=\frac{1}{2} \dot{q}^{\top} C \dot{q}
$$

where $C=C(\theta, \lambda)$ is known as the damping matrix. The structure of $C$ is similar to that of the mass matrix $M(\theta, \lambda)$. An important special case (known as proportional damping; see [25], pg. 140) is when $C$ is a linear combination, $C=\nu M+\nu^{\prime} K$, of the mass matrix $\mathrm{M}$ and of the stiffness matrix $K$ (to be defined below). In principle the parameters $\nu$ and $\nu^{\prime}$ could be inferred from the data. But in our experiments, we take $\nu^{\prime}=0$ and choose $\nu$ judiciously.

Damping forces derived from Rayleigh's dissipation energy have worked well in our experiments. But there are more general forms of damping forces which are nonlinear in velocities, and can still be derived from energy functionals. One such functional that could be useful for some tracking problems and is computationally feasible is that of Lur'e [22]; the damping forces corresponding to this functional are quadratic in the velocities.

Strain Energy. We consider elastic forces that generalize the classical Hooke's law in which (strain) forces are proportional to the displacement. They are derived from a strain energy of the form

$$
U=\frac{1}{2} \int_{V} \epsilon^{\top}(\xi) E \epsilon(\xi) d \xi
$$

where $\epsilon=\left(\epsilon_{11}, \epsilon_{22}, \epsilon_{12}\right)^{\top}$ is a strain vector and $E$ is a $3 \times 3$ matrix known [24,6] as the matrix of elastic (or stiffness) coefficients; the matrix $E$ depends on the material properties of the object. The components of the strain vector $\epsilon$ are the three independent components of the symmetric strain matrix

$$
\hat{\epsilon}=\frac{1}{2}\left(I+J^{\top}+J^{\top} J\right)
$$

where $J=\frac{\partial u}{\partial \xi}$ is the Jacobian matrix of $u(\xi)$ defined in (2.5). The strain energy (3.6) arises from the generalized Hooke's law (which states [24] that the stress vector $\sigma$ is given by $\sigma=E \epsilon$ ). Hooke's law is an approximation (see [6], pg. 250) that typically holds for small strains, i.e., when the $J^{\top} J$ term in $\hat{\epsilon}$ is neglected. Nevertheless, we will use (3.6) even in the presence of this term.

Let $e_{0}(\lambda)$ denote the column vector with entries $\lambda_{i} \lambda_{j}, i, j=1, \ldots, N_{\lambda}$. Using $(2.5 \mathrm{~b})$ one can derive (see Appendix) that 


$$
\epsilon=D_{0}\left((\xi) \lambda+Q_{0}\right)(\xi) e_{0}(\lambda)
$$

where $D_{0}$ and $Q_{0}$ are $3 \times N_{\lambda}$ and $3 \times \frac{N_{\lambda}\left(N_{\lambda}+1\right)}{2}$ matrices, respectively. Then (3.6) reads:

$$
U=\frac{1}{2} \lambda^{\top} K_{1} \lambda+\frac{1}{2} e_{0}^{\top}(\lambda) K_{2}(\lambda)
$$

with

$$
K_{1}=\int_{V} D_{0}^{\top} E D_{0} d \xi \quad, \quad K_{2}=\int_{V} Q_{0}^{\top} E Q_{0} d \xi
$$

Equation (3.8a) may be written in a form more convenient for our equation, i.e.,

$$
U=\frac{1}{2} \tilde{e}^{\top}(\lambda) \tilde{K} \tilde{e}(\lambda)
$$

with

$$
\tilde{K}=\left(\begin{array}{cc}
K_{1} & 0 \\
0 & K_{2}
\end{array}\right), \tilde{e}=\left(\begin{array}{c}
\lambda \\
e_{0}(\lambda)
\end{array}\right)
$$

For small deformations (strains), the second term in (3.8a) is neglected, and the matrix $K_{1}$ is called the stiffness matrix. In our implementations, we keep both terms in (3.8).

The matrix $E$ may depend on $\xi$. In our experiments we take $E$ to be a constant matrix of the form

$$
E=\left(\begin{array}{ccc}
\kappa_{1}+2 \kappa_{2} & \kappa_{1} & 0 \\
\kappa_{1} & \kappa_{1}+2 \kappa_{2} & 0 \\
0 & 0 & 2 \kappa_{2}
\end{array}\right), \text { or } \quad E=\left(\begin{array}{ccc}
\kappa_{1} & 0 & 0 \\
0 & \kappa_{2} & 0 \\
0 & 0 & 0
\end{array}\right)
$$

where the constants $\kappa_{1}$ and $\kappa_{2}$ are analogous to Lame's constants [24, 22]. These type of $E$ 's are appropriate for homogeneous material that exhibit certain symmetries [24]. Some guidelines for choosing the constants $\kappa_{1}$ and $\kappa_{2}$ are given in $\S 6$.

In the case of affine transformations, the strain vector and the strain energy are given by

$$
\begin{gathered}
\epsilon=\left(\lambda_{1}+\frac{\lambda_{1}^{2}+\lambda_{3}^{2}}{2}, \lambda_{2}+\frac{\lambda_{2}^{2}+\lambda_{3}^{2}}{2},\left(1+\frac{\lambda_{1}+\lambda_{2}}{2}\right) \lambda_{3}\right)^{\top} \\
U=\frac{1}{2} \epsilon^{\top}(\lambda) K_{0} \epsilon(\lambda) \quad, \quad K_{0}=\int_{V} E d \xi .
\end{gathered}
$$


3.2. Continuous-Time and Discrete-Time Equations of Motion. Having defined the kinetic, dissipation, and strain energies $T, D$, and $U$, respectively, the equations of motion for $q(t)$ are derived from Lagrange's equations

$$
\frac{d}{d t}\left(\frac{\partial T}{\partial \dot{q}}\right)-\frac{\partial T}{\partial q}+\frac{\partial D}{\partial \dot{q}}+\frac{\partial U}{\partial q}=Q
$$

where $Q$ is a random generalized external force (we shall elaborate on the randomness and nature of $Q$ shortly). A straightforward computation (see Appendix) yields the equation

$$
M(q) \ddot{q}+F(q, \dot{q})+C(q) \dot{q}+B(\lambda) K e(\lambda)=Q,
$$

where $M(q)$ is the mass matrix, $C(q)$ the damping matrix, and $B(\lambda), K$, and $e(\lambda)$ are constructed (see Appendix) in terms of the objects in $(3.9 \mathrm{~b})$. The term $F(q, \dot{q})$ is quadratic in $\dot{q}$ (see Appendix) and corresponds to forces analogous to the gyroscopic and Coriolis forces in 3-dimensions; this term depends only on $\theta, \lambda, \dot{\theta}$, and $\dot{\lambda}$ (it is independent of $\alpha$ and $\dot{\alpha}$ ). Equation (3.11) needs to be accompanied by some initial conditions $q(0)$ and $\dot{q}(0)$.

In the tracking context, one does not observe the system at every instance of time, but only at discrete time intervals $k \Delta t, k=0,1,2, \ldots$, separated by a sampling rate $\Delta t$. Thus we discretize (3.11) as follows: $q_{k}=q(k \Delta t) ; \dot{q}$ and $\ddot{q}$ are approximated by $\left(q_{k}-q k-1\right)(\Delta t)^{-1}$ and $\left(q_{k+1}-2 q_{k}+q_{k-1}\right)(\Delta t)^{-2}$, respectively. Also define $\tilde{W}_{k}=$ $(\Delta t)^{2} Q(k \Delta t)$. Then the discretized version of $(3.11)$ reads

$$
q_{k+1}=\Phi\left(q_{k}, q_{k-1}\right)+M^{-1}\left(q_{k}\right) \tilde{W}_{k} \quad, \quad k=0,1,2, \ldots
$$

where

$$
\begin{aligned}
\Phi\left(q_{k}, q_{k-1}\right)= & 2 q_{k}-q_{k-1}-(\Delta t)^{2} M^{-1}\left(q_{k}\right)\left[F\left(q_{k}, \frac{q_{k}-q_{k-1}}{\Delta t}\right)+\right. \\
& \left.C\left(q_{k}\right) \frac{q_{k}-q_{k-1}}{\Delta t}+B^{\top}\left(q_{k}\right) \operatorname{Ke}\left(q_{k}\right)\right]
\end{aligned}
$$

with the convention $q_{-1}=0$. This may be written in terms of the state vector

$$
X_{k}=\left(\begin{array}{c}
q_{k} \\
q_{k-1}
\end{array}\right) \quad, \quad k \geq 0
$$

as follows:

$$
X_{k+1}=f\left(X_{k}\right)+g\left(X_{k}\right) W_{k} \quad, \quad k \geq 0
$$

where

$$
f\left(X_{k}\right)=\left(\begin{array}{c}
q_{k} \\
\Phi\left(q_{k}, q_{k-1}\right)
\end{array}\right) \quad, \quad g\left(X_{k}\right)=M^{-1}\left(q_{k}\right) \quad, \quad W_{k}=\left(\begin{array}{c}
0 \\
\tilde{W}_{k}
\end{array}\right) .
$$

The external forces acting on the object are typically unknown. For example, a vehicle or an aircraft moves smoothly most of the time, but due to unpredictable effects the direction of motion may change abruptly. This type of uncertainty makes the "external force" or noise $W_{k}, k \geq 0$, random. Randomness in the model also enters through the 
initial condition (3.1b). As in classical filtering applications $[10,9]$, we assume that the $W_{k}$ 's are independent from each other as well as independent of $X_{0}$. If we denote the probability distribution of $W_{k}$ by $p_{w}(\cdot)$, then $X_{k}, k \geq 0$, defined by (3.12), is a Markov chain with transition probabilities given by (3.1a) with $M\left(X_{k}\right)$ the mass matrix, and $\Psi\left(X_{k}\right)=M\left(X_{k}\right) f\left(X_{k}\right)$ where $f\left(X_{k}\right)$ is defined in $(3.12 \mathrm{~b})$. The noise $W_{k}$ need not be Gaussian; in fact in one of our experiments $p_{w}$ is taken to be a mixture of Gaussians (see $\S 6)$. This completes our description of the dynamic model.

4. Data Models. Let $Y_{k}: 0 \leq k \leq T$ be a sequence of observations, and $X_{k}: 0 \leq k \leq T$ a corresponding sequence of state vectors. We will assume that the $Y_{k}$ 's are conditionally independent in the sense that

$$
\prod_{k=0}^{T} P\left(Y_{0}, \ldots, Y_{T} \mid X_{0}, \ldots, X_{T}\right)=\prod_{k=1}^{T} P_{k}\left(Y_{k} \mid X_{k}\right) .
$$

A data (or observation) model is defined by specifying the conditional probability (likelihood function) $P_{k}(y \mid x)=P_{k}\left(Y_{k}=y \mid X_{k}=x\right)$. Equivalently, a data model can be defined by a functional relation of the form

$$
Y_{k}=h_{k}\left(X_{k}, V_{k}\right) \quad, \quad k \geq 0
$$

with some function $h_{k}$, and $V_{k}, k \geq 0$, a sequence of independent noise.

Below we define two data models (Model 1 and Model 2). The first model specifies $P_{k}\left(Y_{k} \mid X_{k}\right)$ while the second model is described in the form (4.2) with $h_{k}$ linear in both $X_{k}$ and $V_{k}$. Model 1 is combined with the Monte Carlo Filter (MCF) tracking algorithm, while Model 2 is combined with the Extended Kalman Filter (EKF). In the first model, $Y_{k}$ represents the raw gray-level data at frame $k$ together with differences between the gray-levels at frame $k$ and a previous frame (the choice of the previous frame depends on the time revolution; in our implementations we use $k-2$ ). In the second model, $Y_{k}$ represents an estimate of the coordinate vector $q_{k}$ at frame $k$, and $h_{k}$ depends only on $q_{k}$. Both models exploit the output of the prediction step in the filtering algorithm (the MCF for Model 1, and EKF for Model 2).

4.1. Data Model 1. Given the state vector $X_{k}$, an instantiation $O_{k}$ of the object is determined at frame $k$ using the first component $q_{k}$ of $X_{k}$ and the template $V$. In the implementations $X_{k}$ represents one of the predictive samples (see $\S 5$ ) of the MCF. Thus $O_{k}$ is a candidate location for the true instantiation of the object. For such a candidate location, we construct a contrast statistic $D\left(Y_{k} ; X_{k}\right)$ by comparing the data inside $O_{k}$ with the data outside $O_{k}$. The statistic $D\left(Y_{k} ; X_{k}\right)$ is large (resp. small) if the data inside and the data outside are very different (resp. very similar), indicating that $O_{k}$ is close (resp. not close) to the true instantiation of the object. More precisely, for an appropriate threshold $D^{*}$, if $D\left(Y_{k} ; X_{k}\right) \gg D^{*}$ then $O_{k}$ is a good candidate location (possibly the true ones), while if $D\left(Y_{k} ; X_{k}\right) \ll D^{*}$ then $O_{k}$ is not a good candidate location.

The precise form of our choice for $D\left(Y_{k} ; X_{k}\right)$ will be given below. But having specified the contrast statistic $D\left(Y_{k} ; X_{k}\right)$, we define a likelihood function by 


$$
P\left(Y_{k} \mid X_{k}\right)=\frac{1}{Z\left(X_{k}\right)} \exp \left\{\varphi\left(D\left(Y_{k} ; X_{k}\right)\right)\right\}
$$

where $Z\left(X_{k}\right)$ is a normalizing constant and $\varphi(\cdot)$ is an appropriate function; examples of $\varphi(\cdot)$ are $\varphi(D)=D$, or

$$
\varphi(D)= \begin{cases}1-\left(\frac{D-D^{*}}{D^{*}}\right)^{2} & \text { for } D \geq D^{*} \\ 1+\left(\frac{D-D^{*}}{D^{*}}\right)^{2} & \text { for } D^{*} \leq D \leq D_{\max }\end{cases}
$$

where $D^{*}$ is a threshold and $D_{\max }$ is a maximum value for $D$. In principle, the threshold $D^{*}$ can be chosen so that the Type I error is small. The above choices of $\varphi$ ensure that the likelihood function is high for $D \gg D^{*}$ and small for $D \ll D^{*}$.

Next we define our contrast statistic $D\left(Y_{k} ; X_{k}\right)$ : we choose a neighborhood $I_{k}$ of the boundary $\partial O_{k}$ in the interior of $O_{k}$, and a neighborhood $E_{k}$ of $\partial O_{k}$ in the exterior of $O_{k}$. We divide $\partial O_{k}$ into $L$ segments, $\partial O_{k, l}, \quad l=1, \ldots, L$ (the segments need not be of equal length. In our implementations, the segments are determined by choosing $L$ equally spared points on the boundary $\partial V$ of the template; then these points and $q_{k}$ determine the segments $\partial O_{k, l}$ ). Each segment $\partial O_{k, l}$ determines an interior neighborhood $I_{k, l} \subset I_{k}$ and an exterior neighborhood $E_{k, l} \subset E_{k}$ by considering the lines vertical to $\partial O_{k}$ at the end points of $\partial O_{k, l}$. Let $Y_{k, l}$ denote the collection of gray-level in $I_{k, l} \cup E_{k, l}$ $(l=1, \ldots, L)$. Comparing the data in $I_{k, l}$ with the data in $E_{k, l}$, we design (see below) a local contrast statistic $D_{k, l}^{(1)}\left(Y_{k, l} ; X_{k}\right)$; then we augment (see below) this statistic using the differences of the gray-levels at frame $k$ and a previous frame (say $k-2$ ). Let $D_{k, l}$ denote the augmented statistic. Then, our overall contrast statistic is defined by

$$
D\left(Y_{k} ; X_{k}\right)=\sum_{l=1}^{L} D_{k, l}
$$

Next we define the local statistic $D_{k, l}^{(1)}\left(Y_{k, l} ; X_{k}\right)$ and its augmentation $D_{k, l}$. There are various ways of comparing the data in $I_{k, l}$ and $E_{k, l}$. The simplest (and the one we use in our experiments of $\S 6$ ) is the following: let $m_{I_{k, l}}, m_{E_{k, l}}$ denote the means of the gray-levels in the neighborhoods $I_{k, l}$ and $E_{k, l}$, respectively. Also, let

$$
s_{k, l}^{2}=\frac{1}{\left|I_{k, l}\right|+\left|E_{k, l}\right|}\left\{\sum_{i \in I_{k, l}}\left(Y_{k}^{(i)}-m_{I_{k, l}}\right)^{2}+\sum_{i \in E_{k, l}}\left(Y_{k}^{(i)}-m_{E_{k, l}}\right)^{2}\right\},
$$

where $Y_{k}^{(i)}$ denote the image intensity at pixel $i$. Then the local contrast statistic is defined by

$$
D_{k, l}^{(1)}=D_{k, l}^{(1)}\left(Y_{k, l} ; X_{k}\right)=\frac{\left|m_{I_{k, l}}-m_{E_{k, l}}\right|}{s_{k, l}} .
$$

This simple statistic (based on means and variance) has worked well in our experiments. More sophisticated contrast statistics can be designed in terms of rank based statistics [12] 
such as the Mann-Witney and the Smirnov-Kolmogorov statistics. These "distributionfree" statistics have the advantage of being invariant under certain monotone transformation of the data. In our experiments these statistics have given comparable results with the above mean and variance statistic, but they might be useful in other tracking environments.

The splitting of the boundary into segments and the introduction of local contrast statistics is significant in capturing variations in lighting conditions around the boundary. For example, if the object is brightly illuminated from above and shadowed below, the gray-level values in the interior of the object will be significantly different near the top and bottom. These regions, therefore, need to be treated separately if mean-based statistics (such as the ones in (4.5)) are to be used.

The augmentation of $D_{k, l}^{(1)}$ is defined as follows: let $\tilde{y}_{k}$ denote the differences between the gray-levels at frame $k$ and a previous frame (say $k-2$ ); moreover, let $\tilde{y}_{k, l}$ denote the differences in $I_{k, l} \cup E_{k, l}$. We define a new local contrast statistic $D_{k, l}^{(2)}\left(\tilde{y}_{k, l} ; X_{k}\right)$ the same way we defined $D_{k, l}^{(1)}$, except that $Y_{k, l}$ is replaced by $\tilde{y}_{k, l}$. Then we define a weighting factor

$$
\gamma_{k, l}=\gamma-(\gamma-1) e^{-\mu D_{k, l}^{(2)}}
$$

with some constants $\gamma>1$ and $\mu>0$. Note that $\gamma_{k, l} \simeq 1$ when $D_{k, l}^{(2)}$ is very small, and $\gamma_{k, l} \simeq \gamma$ when $D_{k, l}^{(2)}$ is very large. The exact meaning of "very small" and "very large" depends on the constant $\mu$ (both $\gamma$ and $\mu$ are chosen judiciously in our experiments). Then the augmented local contrast statistic $D_{k, l}$ is defined by

$$
D_{k, l}=\gamma_{k, l} D_{k, l}^{(1)}
$$

The data model defined by the likelihood function (4.3) leads to a fundamental difficulty: the normalizing constant $Z\left(X_{k}\right)$ in $(4.3)$ is practically uncomputable. Consequently the implementation of the Monte Carlo Filter algorithm is computationally unfeasible. However, in $\S 5$ we explore the structure of the MCF (especially its update step), and introduce a modification of the model (4.3) that makes computation feasible (in fact, tracking runs in real-time).

4.2. Data Model 2. Our second data model is defined by a functional relation of the form (4.2) with $h_{k}$ depending only on $q_{k}$ and being linear on both $q_{k}$ and $V_{k}$. As we mentioned before, Model 2 is combined with EKF. The observation $Y_{k}$ in this model is an estimate of the generalized coordinates $q_{k}$ at frame $k$. The estimate $Y_{k}$ of $q_{k}$ involves two major components: first, a segmentation algorithm whose output is a set of points $\hat{x}_{k}^{(l)}, l=1, \ldots, L$ assumed to be on the boundary of the estimated instantiation of the object; the segmentation employs the gray-level data at frame $k$, gray-level differences between frames $k$ and $k-2$, and the output of the prediction step of the EKF. The second component consists in going from $\left\{\hat{x}_{k}^{(l)}: l=1, \ldots, L\right\}$ to $Y_{k}$. This component is described first. For ease of notation we suppress the frame index $k$.

To estimate $q=(\alpha, \theta, \lambda)^{\top}$ on the basis of $\left\{\hat{x}^{(l)}: l=1, \ldots, L\right\}$, we use the alternative parametrization given by $(2.2 \mathrm{a})$ and $(2.3)$. Let $b=(\alpha, a)^{\top}$, where $a$ is a vector representing the parameters in the matrices $A_{m}, m=1, \ldots, n$. First, we derive an estimate 
$\hat{b}$ of $b$ (on the basis of $\left.\left\{\hat{x}^{(l)}: l=1, \ldots, L\right\}\right)$. Then an estimate of $\theta$ is given by

$$
\tan \hat{\theta}=\frac{\hat{a}_{11}-\hat{a}_{12}}{\hat{a}_{11}+\hat{a}_{22}}
$$

where $\left\{\hat{a}_{i j}: i, j=1,2\right\}$ is an estimate of the matrix elements of $A_{1}$. An estimate $\hat{\lambda}$ of $\lambda$ is obtained by the defining equations (see below (2.4)) for $\Lambda_{m}, m=1, \ldots, M$.

The estimate $\hat{b}$ of $b$ is obtained as follows. Let $\left\{\xi^{(l)}: l=1, \ldots, L\right\}$ be $\mathrm{L}$ points on the boundary $\partial V$ of the template $V$, and define

$$
\mathcal{E}\left(b ; \xi^{(1)}, \ldots, \xi^{(L)}\right)=\sum_{l=1}^{L}\left|\hat{x}^{(l)}-\alpha-\sum_{j=1}^{n} A_{j} e_{j}\left(\xi^{(l)}\right)\right|^{2}
$$

Then choose $\hat{b}$ and $\hat{\xi}^{(l)}, l=1, \ldots, L$ so that

$$
\mathcal{E}\left(\hat{b} ; \hat{\xi}^{(l)}, \ldots, \hat{\xi}^{(L)}\right)=\min _{b} \min _{\xi(l)} \mathcal{E}\left(b ; \xi^{(l)}, \ldots, \xi^{(L)}\right)
$$

This is a highly non-trivial optimization problem (it is nonlinear even in the affine case $\left.A_{2}=\ldots=A_{n}=0\right)$. We solve this problem by the following greedy algorithm: let $\tilde{b}$ (equivalently, $\tilde{q}$ ) be the predictive estimate (at the current frame) of the EKF algorithm, and for convenience set $\Xi=\left(\xi^{(1)}, \ldots, \xi^{(L)}\right)$; then the steps of the greedy algorithm are:

(1) Start with the initial parameter set $b^{(0)}=\tilde{b}$;

(2) With $b$ fixed to $b^{(0)}$, minimize $\mathcal{E}\left(b^{(0)} ; \Xi\right)$ with respect to $\Xi$ to get $\Xi^{(0)}$;

(3) With $\Xi$ fixed to $\Xi^{(0)}$, minimize $\mathcal{E}\left(b ; \Xi^{(0)}\right)$ to get $b^{(1)}$;

(4) Repeat steps (2) and (3) iteratively to obtain an estimate $b^{(n)}$ of $b$ (and $\Xi^{(n)}$ of $\Xi$ ) at stage $n$; stop when the difference in successive estimates of $b$ is below a certain threshold.

The estimate $\hat{b}$ given by this algorithm is sub-optimal. Nevertheless, the algorithm has been found to work well in our experiments.

Next we turn to the segmentation algorithm for obtaining the points $\hat{x}^{(l)}, l=1, \ldots, L$. In principle, any segmentation algorithm could be adopted for this task. We employ a segmentation algorithm based on the hierarchical/syntactic representation for starshaped objects described in $§ 2.2$; the structure of this representation allows dynamic programming type computations for attaining segmentation. We recall that a star-shaped object is represented by a binary tree (see Fig. 2.1) with terminal nodes indexed by $Z_{N}=\left\{Z_{N, k}: k=0,1, \ldots, 2^{N}-1\right\}$, and intermediate nodes by $Z_{n, k}, k=0,1, \ldots, 2^{n}-1$, $n=0,1, \ldots, N-1$. In this representation, the object is approximated by a polygon with $2^{N}$ vertices. The vertices of our estimated polygon will be the points $\hat{x}^{(l)}, l=1, \ldots, L$ $\left(L=2^{N}\right)$.

A terminal node $Z_{N, k}$ represents a triangle in the polygonal approximation (see Fig. 4.1). As in our description of the first data model, we consider interior and exterior neighborhoods $I$ and $E$ (see Fig. 4.1) about the boundary segment of the triangle. As in $\S 4.1$, we define a local contrast statistic $D\left(Z_{N, k}\right)$ 


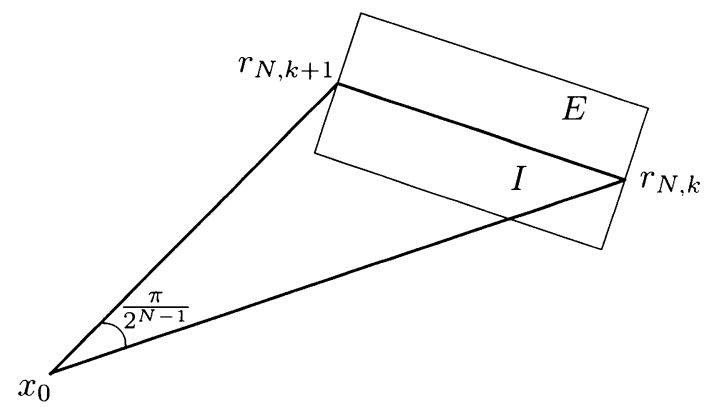

FIG. 4.1. A triangle in the polygonal approximation and neighborhoods $I$ and $E$.

$$
D\left(Z_{N}\right)=\sum_{k=0}^{2^{N}-1} D\left(Z_{N, k}\right) .
$$

The vertices of the polygon are determined by maximizing this contrast statistic. This maximization can be attained via dynamic programming by exploring the tree representation of our object. Recalling the "production rule" (2.7), we define iteratively a contrast statistic for each non-terminal state $Z_{n, k}$ :

$$
D\left(Z_{n, k}\right)=\max _{r_{n+1,2 k+1}}\left\{D\left(Z_{n+1,2 k}\right)+D\left(Z_{n+1,2 k+1}\right)\right\} \quad, \quad k=0, \ldots, 2^{n}-1 .
$$

Thus, starting with the contrast statistics at level $N$, we may inductively calculate the contrast statistics for all intermediate nodes back to the root node $Z_{00}$. This gives the dynamic programming procedure for finding the contrast statistic for the start state $Z_{00}$, corresponding to the optimal tree (or "derivation" tree in the language of context-freegrammars). The steps of the segmentation algorithm may be summarized as follows:

1. Determine $D\left(Z_{N, k}\right), k=0,1, \ldots, 2^{N}-1$. Set $n=N-1$;

2. For each $k=0, \ldots, 2^{n}-1$, and for each $Z_{n, k}$ :

i. For each $r_{n+1,2 k+1}$, evaluate

$$
D\left(Z_{n+1,2 k}\right)+D\left(Z_{n+1,2 k+1}\right)
$$

ii. Determine the maximal contrast statistic $D\left(Z_{n, k}\right)$ using (4.9). Record the value $r_{n+1,2 k+1}$ for the optimal production.

3. Repeat step 2 for $n=N-2, \ldots, 0$, to obtain the optimal contrast statistic for the root node $Z_{00}$;

4. Determine the optimal polygon by applying the optimal production in the order $n=0,1, \ldots, N-1$.

The main computational cost is in step 2. If the range of $r$ is quantized to $N_{r}$ values, then the computational cost of step 2 is $O\left(2^{n} N_{r}^{3}\right)$. Thus the overall computational cost is $O\left(\left(\sum_{n=0}^{N-1} 2^{n}\right) N_{r}^{3}\right)=O\left(2^{N} N_{r}^{3}\right)$. 
To complete our segmentation algorithm, we need to locate a point $x_{0}$ in the image (at the current frame) so that the expected instantiation of the object is star-shaped with respect to $x_{0}$. We refer to $x_{0}$ as the point of initialization (at the current frame). To locate this point we use two types of information: first, given the initialization point at the previous frame, the prediction step of the EKF gives the "predictive" location of the initialization point at the current frame. Often, this predictive initialization point lies at or near a true initialization point. Second, we perform a background substraction, or consider differences in image intensities between the current frame and one or two previous frames. Then we perform a local search to find a point of initialization. A number of even spaced pixels in the vicinity of the dynamic prediction are sampled and the pixel of the highest magnitude is used as an initialization point. Our experiments demonstrate that this heuristic procedure is an effective method for detecting points of initialization.

5. Nonlinear Filtering Algorithms. The tracking problem amounts to solving the nonlinear filtering problem of $(3.12 \mathrm{a}),(3.1 \mathrm{~b})$, and (4.2), which we cast in a slightly more general form

$$
\begin{aligned}
X_{k+1} & =F_{k}\left(X_{k}, W_{k}\right), k \geq 0, \\
X_{0} & \sim P\left(X_{0}\right), \\
Y_{k} & =h_{k}\left(X_{k}, V_{k}\right),
\end{aligned}
$$

with the assumption that $\left\{W_{k}: k \geq 0\right\}$ is a sequence of independent random vectors, as is the sequence $\left\{V_{k}: k \geq 0\right\}$; both sequences are assumed to be independent of each other and of the initial condition $X_{0}$.

Under the above assumptions, the nonlinear filtering problem (5.1) is equivalent to a Hidden Markov Model (HMM): $\left\{X_{k}: k \geq 0\right\}$ is a Markov Chain

$$
P\left(X_{0}, X_{1}, \ldots, X_{T}\right)=P\left(X_{0}\right) \prod_{k=0}^{T-1} P_{k}\left(X_{k+1} \mid X_{k}\right)
$$

and the data $\left\{Y_{k}: k \geq 0\right\}$ satisfy (4.1). In our case, the transition probabilities $P_{k}\left(X_{k+1} \mid X_{k}\right)$ are independent of $k$, but the likelihood function $P_{k}\left(Y_{k}=y \mid X_{k}=x\right)$ depends on $k$. In the following, we shall suppress the $k$-dependence both for the transition probabilities and the likelihood function.

The main object of interest in any filtering algorithm is the posterior distribution $P\left(X_{k} \mid Y_{0}, \ldots, Y_{k}\right), k \geq 0$. But the predictive distribution $P\left(X_{k} \mid Y_{0}, \ldots, Y_{k-1}\right), k \geq 0$ (with the convention $P\left(X_{0} \mid Y_{-1}\right)=P\left(X_{0}\right)$ ) also plays a fundamental role. From (5.2) and (4.1), one easily shows

$$
P\left(X_{k} \mid Y_{0}, \ldots, Y_{k-1}\right)=\int P\left(X_{k} X_{k-1}\right) P\left(X_{k-1} \mid Y_{0}, \ldots, Y_{k-1}\right) d X_{k-1}, k \geq 0
$$




$$
P\left(X_{k} \mid Y_{0}, \ldots, Y_{k}\right)=\frac{P\left(Y_{k} X_{k}\right)}{P\left(Y_{k} \mid Y_{0}, \ldots, Y_{k-1}\right)} P\left(X_{k} \mid Y_{0}, \ldots, Y_{k-1}\right) \quad, \quad k \geq 0
$$

These formulas form the basis of the Monte Carlo Filter (MCF) - as well as of any iterative filtering algorithm. Note that (5.3a) relates the predictive distribution at frame $k$ to the posterior distribution at frame $k-1$ (via the dynamics $P\left(X_{k+1}\left(X_{k}\right)\right.$ ), while $(5.3 \mathrm{~b})$ relates the posterior distribution and the predictive at frame $k$ (via the data model $\left.P\left(Y_{k} \mid X_{k}\right)\right)$. An iterative filtering algorithm has two basic steps: a predictive step, in which the solution at frame $k-1$ is propagated to the next frame $k$ via (5.3a) (equivalently, via the dynamics (5.1a)); and an update step, in which the predictive solution is updated by using the data at the current frame $k$.

In all our experiments we solve the nonlinear filtering (5.1) with two algorithms- -the Monte Carlo Filter (MCF) and the Extended Kalman Filter (EKF) - and compare their performances (see $\S 6$ ). Below we describe the $\mathrm{MCF}$, and a modification of our data model (4.3) that makes computations feasible. The EKF is well-known $[1,10]$ and it will not be described here.

The Monte Carlo Filter (MCF). The MCF is a simulation procedure that iteratively generates samples which are (approximately) governed by the posterior distribution $P\left(X_{k} \mid Y_{0}, \ldots, Y_{k}\right), k \geq 0$. There exists, by now, an extensive literature $[13,3,17,7,8]$ on simulation based filters known as Bayesian bootstrap, condensation, particle filters, etc.; they are all related to the Sampling Importance Resampling procedure [23]. Our presentation here is closer to that of [13] which appears to be the first paper where the basic algorithm was introduced (see [7] for a readable account of the procedure and modifications that improve performance and reduce computational cost).

The basic MCF procedure is as follows: suppose that at frame $k-1$, we have $N$ samples $\left\{\hat{X}_{k-1}^{(n)}: n=1, \ldots, N\right\}$ governed (approximately) by the posterior distribution $P\left(X_{k-1} \mid Y_{0}, \ldots, Y_{k-1}\right)$. Then the MCF generates samples $\left\{\hat{X}_{k}^{(n)}: n=1, \ldots, N\right\}$ governed (approximately) by $P\left(X_{k} \mid Y_{0}, \ldots, Y_{k}\right)$, via two steps:

Prediction Step: Generate $N$ independent samples $W_{k-1}^{(n)}, n=1, \ldots, N$ from $P_{k}\left(W_{k}\right)$, and compute

$$
\tilde{X}_{k}^{(n)}=f_{k-1}\left(\hat{X}_{k-1}^{(n)}, W_{k-1}^{(n)}\right), n=1,2, \ldots, N .
$$

This is equivalent to generating $\tilde{X}_{k}^{(n)}$ from the transition probability $P\left(X_{k} \mid \hat{X}_{k-1}^{(n)}\right)$, $n=1, \ldots, N$ (see (5.3a)). It is easily shown that each $\tilde{X}_{k}^{(n)}$ is governed (approximately) by the predictive distribution $P\left(X_{k} \mid Y_{o}, \ldots, Y_{k-1}\right)$. The samples $\tilde{X}_{k}^{(n)}$, $n=1,2, \ldots, N$ will be referred to as the predictive samples.

Update Step: Using the data model $P\left(Y_{k} \mid X_{k}\right)$, define

$$
q_{k}^{(n)}=\frac{P\left(Y_{k} \mid \tilde{X}_{n}^{(n)}\right)}{\sum_{m=1}^{N} P\left(Y_{k} \mid \tilde{X}_{k}^{(m)}\right)}, n=1, \ldots, N .
$$

Then view $q_{k}=\left(q_{k}^{(1)}, \ldots, q_{k}^{(N)}\right)$ as a vector of probability weights on the set of predictive samples $\left(\tilde{X}_{k}^{(1)}, \ldots, \tilde{X}_{k}^{(N)}\right)$. Sample $N$ times, independently with 
replacement, from $\left\{\tilde{X}_{k}^{(n)}\right\}$ with probability weights $\left\{q_{k}^{(n)}\right\}$ to obtain $\hat{X}_{k}^{(n)}, n=$ $1,2, \ldots, N$. Using $(5.3 \mathrm{~b})$, it is easily shown that $\left\{\hat{X}_{k}^{(n)}\right\}$ are independent samples governed (approximately) by $P\left(X_{k} \mid Y_{0}, \ldots, Y_{k}\right)$. There are fast algorithms [17, 7] for the above resampling step, i.e., for generating $\left\{\hat{X}_{k}^{(n)}\right\}$ from $\left\{\tilde{X}_{k}^{(n)}\right\}$. The samples $\left\{X_{k}^{(n)}\right\}$ will be referred to as the posterior samples.

The posterior distribution $P\left(X_{k} \mid Y_{0}, \ldots, Y_{k}\right)$ can be approximated either by the equally weighted posterior samples $\left\{\hat{X}_{k}^{(n)}: n=1, \ldots, N\right\}$ or by the weighted predictive samples $\left\{\left(\tilde{X}_{k}^{(n)}, q_{k}^{(n)}\right): n=1, \ldots, N\right\}$. For example, if we want to estimate the posterior mean

$$
\Theta_{k}=\int \phi\left(X_{k}\right) P\left(X_{k} \mid Y_{0}, \ldots, Y_{k}\right) d X_{k}
$$

of a function $\phi(\cdot)$, then

$$
\hat{\Theta}_{k}=\frac{1}{N} \sum_{n=1}^{N} \phi\left(X_{k}^{(n)}\right)
$$

and

$$
\tilde{\Theta}_{k}=\sum_{k=1}^{N} \phi\left(\tilde{X}_{k}^{(n)}\right) q_{k}^{(n)}
$$

are both consistent estimators of $\Theta_{k}$. However, it can be shown [7] that $\tilde{\Theta}_{k}$ is a better estimator in the sense that its variance is smaller than that of $\hat{\Theta}_{k}$. Similarly, the state vector $X_{k}$ at frame $k$ can be estimated either in terms of the posterior samples $\left\{\hat{X}_{k}^{(n)}\right\}$ or in terms of the weighted predictive samples $\left\{\left(\tilde{X}_{k}^{(n)}, q_{k}^{(n)}\right): n=1, \ldots, N\right\}$. The estimate $X_{k}^{*}$ of $X_{k}$ can be taken to be the mean, median, or some other statistic of these samples. In our experiments, $X_{k}^{*}$ is taken to be the median of the posterior samples $\left\{\hat{X}_{k}^{(n)}: n=1, \ldots, N\right\}$.

The main potential weakness of the MCF is the possibility of sample size diminishing. That is, during the update step, the resampling with replacement of $\left\{\tilde{X}_{k}^{(n)}\right\}$ may pick the same sample more than once. This occurs, for example, when the overlap between the mass of $P\left(X_{k} \mid Y_{0}, \ldots, Y_{k-1}\right)$ and $P\left(Y_{k} \mid X_{k}\right)$ (as a function of $\left.X_{k}\right)$ is small; in this case, only a few of the weights will be significant, and so the update step will select the predictive samples corresponding to the high weights more than once. If this occurs in a number of consecutive frames, eventually the number of distinct samples in $\left(\hat{X}_{k}^{(1)}, \ldots, \hat{X}_{k}^{(N)}\right)$ will be small, and the posterior distribution will be poorly approximated. In $[13,7]$, some general procedures are recommended for alleviating this difficulty. In computer vision tracking problems, the severity of the sample size diminishing problem depends on how good the data and dynamic models are. In our experiments, the sample size reduction seems to be minimal even in situations with high clutter and occlusion.

We end this section with a modification of our basic data model (4.3)-(4.6). To bypass the computational difficulty that stems from the uncomputability of the normalizing constant $Z\left(X_{k}\right)$ in (4.3), we observe that the implementation of the MCF does not use the data model $P\left(Y_{k}, X_{k}\right)$ itself, but only the weights $q_{k}^{(n)}$ defined by (5.5). Thus to implement the MCF one can model $q_{k}^{(n)}$ directly. Using the contrast statistic $D\left(Y_{k}, X_{k}\right)$ of $\S 4.1$, we define a new set of weights 


$$
\alpha_{k}^{(n)}=\frac{D\left(Y_{k} ; \tilde{X}_{k}^{(n)}\right)}{\sum_{m=1}^{N} D\left(Y_{k} ; \tilde{X}_{k}^{(m)}\right)}, n=1, \ldots, N .
$$

The qualitative properties of $\alpha_{k}=\left\{\alpha_{k}^{(n)}: n=1, \ldots, N\right\}$ are similar to those of $q_{k}=$ $\left\{q_{k}^{(n)}\right\}$; i.e., a predictive sample has a high weight under $q_{k}$ if and only if it has a high weight under $\alpha_{k}$. In contrast to $\left\{q_{k}^{(n)}\right\}$, the weights $\left\{\alpha_{k}^{(n)}\right\}$ are easy to compute. In our experiments we use the weights $\left\{\alpha_{k}^{(n)}\right\}$.

6. Experiments. In this section we present a range of tracking experiments with real image sequences, using the models and algorithms described in previous sections. In each experiment results are presented for both the Monte Carlo Filter (MCF) and the Extended Kalman Filter (EKF), and their performances are compared. As stated in the Introduction $(\S 1)$, the experiments demonstrate that the MCF is more robust than the EKF in situations with considerable clutter and/or occlusion, but the MCF was generally found to be somewhat less accurate than the EKF in situations where the object was less occluded. A lesson that emerges from our experiments is that if the dynamic and data models are carefully designed, even a simple algorithm such as EKF can achieve good results in relatively complex environments. Our studies also demonstrate that a nonGaussian dynamic model is important in situations where the trajectories have abrupt discontinuities in direction. These properties are demonstrated both by the experiments reported in this section, as well as by simulation experiments (not reported here) where the dynamic model used to simulate data was also used for tracking.

We present two classes of image sequences - one consisting of fish moving in an aquarium, and one of balls on a pool table. Several image sequences are shown for each class, each presenting different difficulties. In each case we describe the main difficulties present in the problem and compare the performances of MCF and EKF. For each class of experiments, we also describe our choices of the template and its deformations, as well as the choices of the parameters that enter in the dynamic and data models.

6.1. Tracking Fish in an Aquarium. We present three different image sequences ("clips") of increasing complexity. In all cases we track a single fish, but other fish (of somewhat different shape) are present in some of the clips. In all clips, the background is highly cluttered, and the resolution of the fish is generally poor (i.e., the size of the fish relative to the image size is small). Lighting conditions vary from clip to clip, and in all cases the illumination of the fish changes dramatically depending on its position; at times the fish is clearly visible, and at times almost invisible. In some cases, the tracked fish becomes partially or totally occluded for short period of times either by background objects or by other fish. In one of the clips the fish goes through a stream of bubbles, and in another the quality of the images was deliberately degraded by spraying a mist of water on the aquarium glass.

The template of the fish corresponds to an equilibrium or average fish position, and was approximated by an ellipse with horizontal axis length 0.3 and vertical axis length 0.1 (in units of image dimension $[-5,5] \times[-3.748,3.748]$ ); the elliptical approximation of the average pose of the particular fish we want to track was quite good everywhere except 
at the tail. The transformations of the template were taken to be a reduced set of affine transformations; i.e., the scale parameter $\lambda_{3}$ was set equal to zero in all frames. Thus the generalized coordinate vector $q$ has five coordinates-two for location $\left(\alpha_{1}, \alpha_{2}\right)$, one for rotation $(\theta)$, and two for scale $\left(\lambda_{1}, \lambda_{2}\right)$; the scale parameter $\lambda_{1}$ amount for rotations of the fish about a vertical axis, while $\lambda_{2}$ amounts for rotation of the fish about a horizontal axis.

The mass matrix was computed by assuming $\rho(\xi) \equiv 1$, and the dumping matrix $C(q)$ was taken to be half of $M(q)$. The strain energy is given by (3.10), and the "stiffness" matrix $K_{0}$ was chosen to be of the form

$$
K_{0}=\left(\begin{array}{cc}
\kappa_{1} & 0 \\
0 & \kappa_{2}
\end{array}\right)
$$

Our choices of $\kappa_{1}$ and $\kappa_{2}$ were motivated by the aspects of the fish motion modeled by the scale parameters $\lambda_{1}$ and $\lambda_{2}$. As mentioned above, $\lambda_{1}$ corresponds to a rotation of the fish about a vertical axis; as the fish turns to face the camera (or turns away from it), the horizontal dimension of the ellipse changes; we expect this type of horizontal scale change to be relatively common, as the fish commonly changes direction swimming from side to side. Accordingly, we choose $\kappa_{1}$ to be very low so as not to inhibit this type of motion. We choose $\kappa_{2}$ to be larger than $\kappa_{1}$. The rational for this is that changes in vertical scale (described by $\lambda_{2}$ and corresponding to a rotation of the fish about a horizontal axis in effect, the fish rolling over on its side) are uncommon; we expect a certain amount of (vertical) scaling due to fish swimming closer and further away from the camera, but we wish to inhibit excessive deformations in the vertical direction. In our examples, we choose $\kappa_{1}=0.1, \kappa_{2}=2$.

Finally, we must specify the noise characteristics in the dynamic and data models. For EKF both $W_{k}$ and $V_{k}$ need to be Gaussian. For $W_{k}$ (dynamic noise) this is not an unreasonable assumption, as the fish moves relatively slowly and no highly abrupt changes of direction occur. Thus for EKF and MCF $W_{k}$ was taken to be Gaussian with mean zero and variance one. For EKF, we must also specify $V_{k}$ (the data model for MCF is determined by (4.8) and (5.6)). Given that the images contain distortion and clutter due to other moving objects, we chose the variance of $V_{k}$ to be much higher than that of $W_{k}$ (in our examples $V_{k}$ has mean zero and variance 5). This choice implies that the evolution of the EKF is primarily determined by the dynamics, with the observation model having only gradual effect; implications of this are discussed later.

For the MCF, we experimented with various sample sizes. The minimal number of samples needed for successful tracking was 500 - this was the number of samples used in the results reported here.

Clip One (Figures 6.1-6.3). In this sequence, the fish was swimming (see Fig. 6.1) from top left of the scene to the center, and then back to the bottom-left. The length of the clip was 720 frames (about 12 seconds). This was intended to be the simplest of the fish sequences. The main difficulty occurred when the fish changed direction (see Fig. 6.1 , \#2 and \#3). For a period of about 8 frames, the fish was facing the camera directly, and its resolution became very poor (see Fig. 6.2). The reader may have some difficulty 


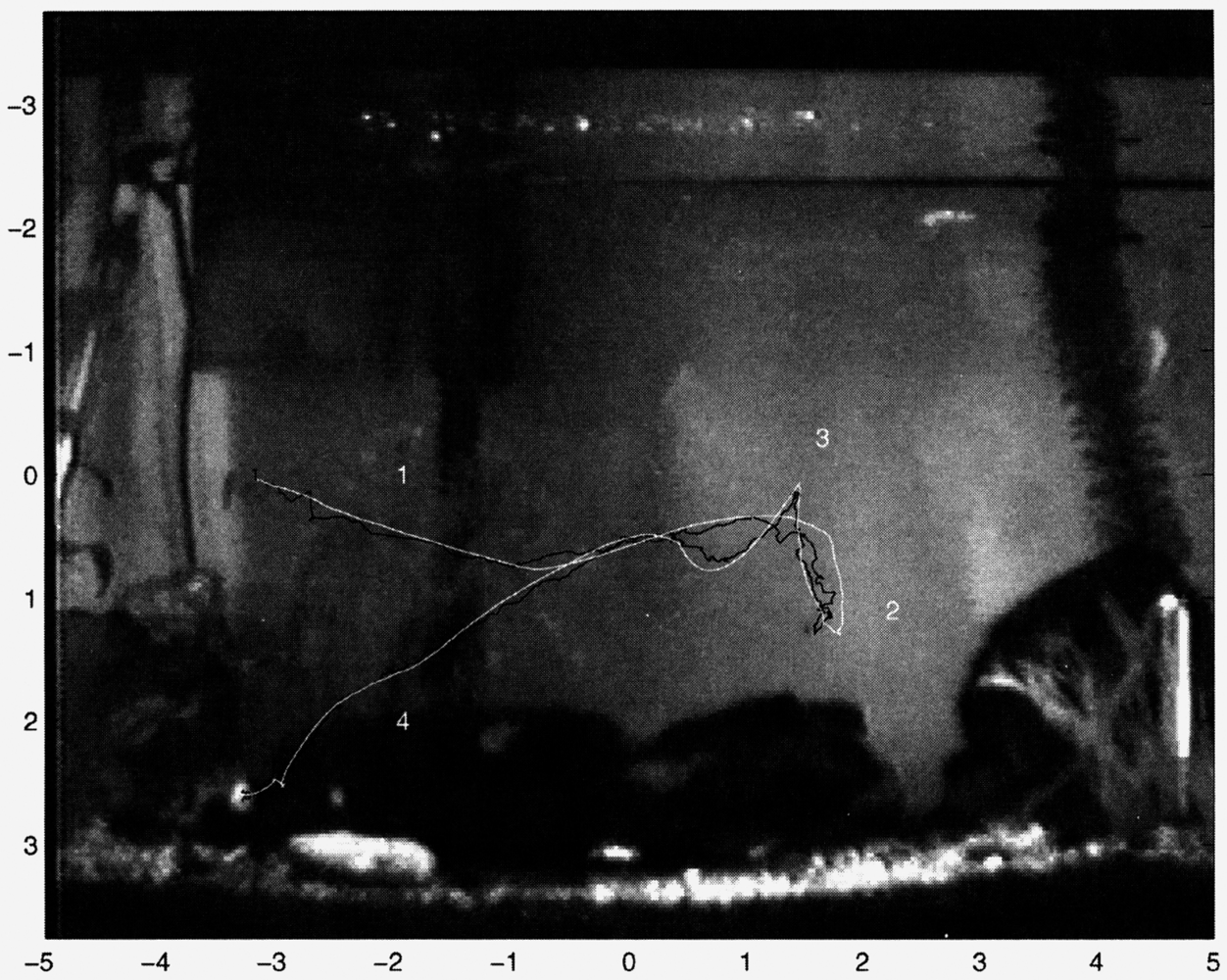

FIG. 6.1. Last frame of Clip One, with estimated trajectories based on the EKF (white curve) and MCF tracker (black curve).

locating the fish in the frame; it is located in the center-right region of the frame, and it appears as a little dark spot. In this frame the ellipse for the fish was placed on a rock.

Figure 6.1 shows the estimated trajectories of the MCF (black curve) and the EKF (white curve). With the exception when the fish was facing the camera (\#2 and \#3), both trackers do a very good job. In regions \#1 and \#4, the fish was relatively well-lit and visible, and the performance of both algorithms is best there.

Figure 6.3 shows the MCF (solid lines) and the EKF (dotted lines) estimates of the generalized coordinates $\alpha_{1}, \alpha_{2}, \theta, \lambda_{1}, \lambda_{2}$. The estimates of $\alpha_{1}$ and $\alpha_{2}$ are relatively smooth; on the other hand, the estimates of $\theta, \lambda_{1}$, and $\lambda_{2}$ are more noisy, though EKF generally does better job then MCF. The variations are larger at points where the resolution of the fish is low, and the lighting conditions create shadows. Both Fig. 6.1 and Fig. 6.3 demonstrate that the EKF estimates of $\alpha_{1}$ and $\alpha_{2}$ are smoother than those of the MCF. This is due to the interplay between the dynamic and the data models. For the EKF, tracking is dominated by the dynamics as the noise variance of the data model was larger than that of the dynamic model. On the other hand, variations in the measurement process are more likely to cause fluctuations in the MCF estimate.

Clip Two (Figure 6.4). In this sequence (1200 frames-approximately 20 seconds) a stream of bubbles was switched on, and the fish being tracked crossed the stream twice 


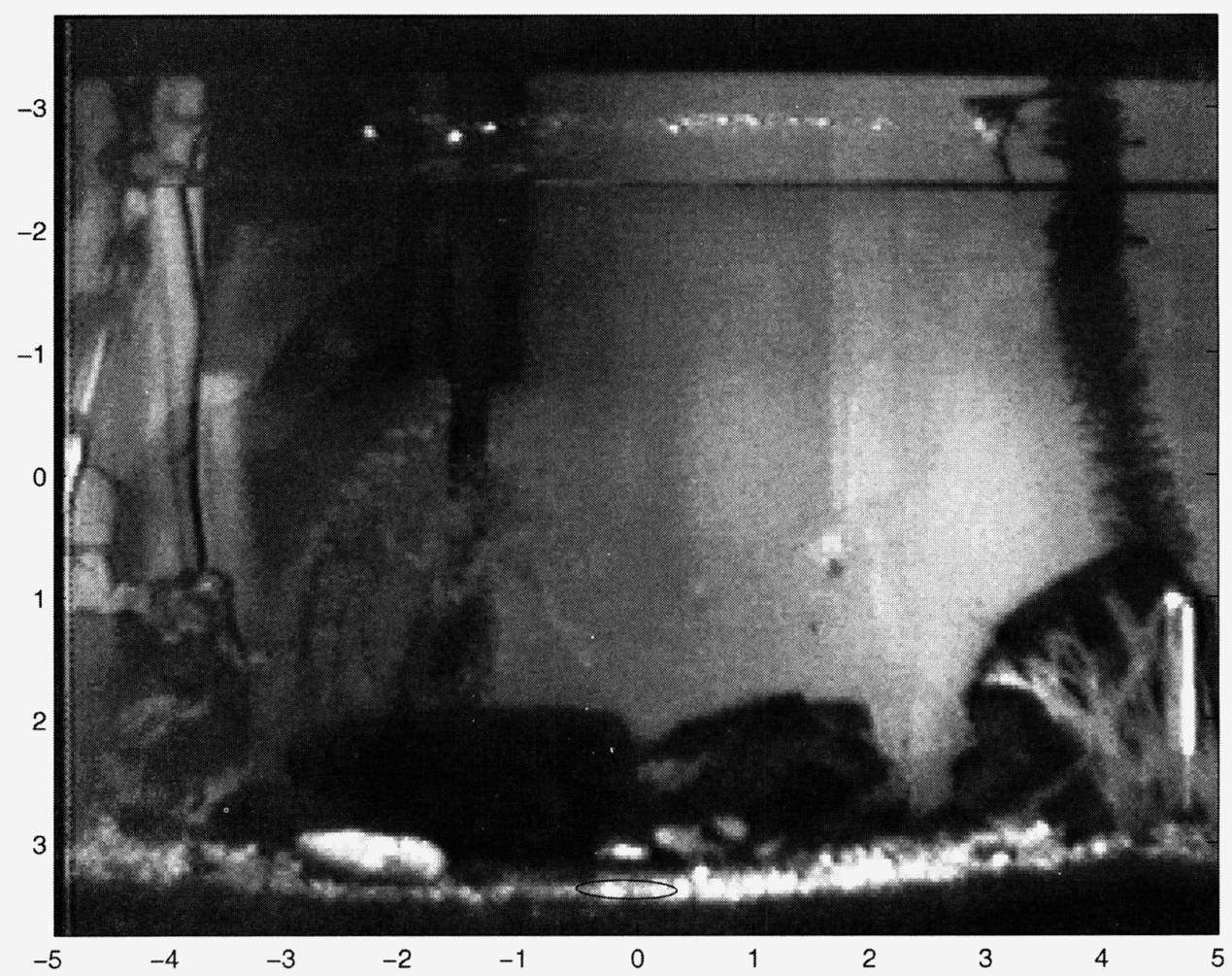

FIG. 6.2. A Clip One frame with very low resolution of the fish (little dark spot at the center-right region of the frame).

(see Fig. 6.4, panels B and C). At some point (to the left of \#2 in panel C) the tracked fish passed near another fish. Panel A shows the first part (up to the point the fish entered the bubble stream) of the EKF estimated trajectory. Panels B and C show the estimated trajectories of the EKF and the MCF, respectively. Clearly, both trackers were quite successful. Again, one observes that the EKF trajectory is smoother than the MCF trajectory. And the reason is the same as for the experiment in Clip One: the dynamic variance is low relative to that of the data model, and so the dynamic model has greater weight in the trajectory estimation. Inside the bubble region, the MCF trajectory is more noisy, and near \#3 (panel C) there is a loss of lock for a short period (the MCF tracker locks on the bubbles); however, the MCF tracker recovers quickly and locks again on the fish (the recovery is helped by the fact that the bubbles are transient - typically persisting for at most couple of frames). While the EKF trajectory is smooth (at least relative to the $\mathrm{MCF}$ ) in the bubble region, the error in the estimation of the shape parameters $\theta, \lambda_{1}$, and $\lambda_{2}$ was relatively large; this is evident from panel $\mathrm{A}$ - the ellipse is somewhat off the fish.

The presence of the second fish (near the region at the left of \#2 in panel C) had a larger effect on the EKF than on the MCF. This is evident from the bump in panel B 

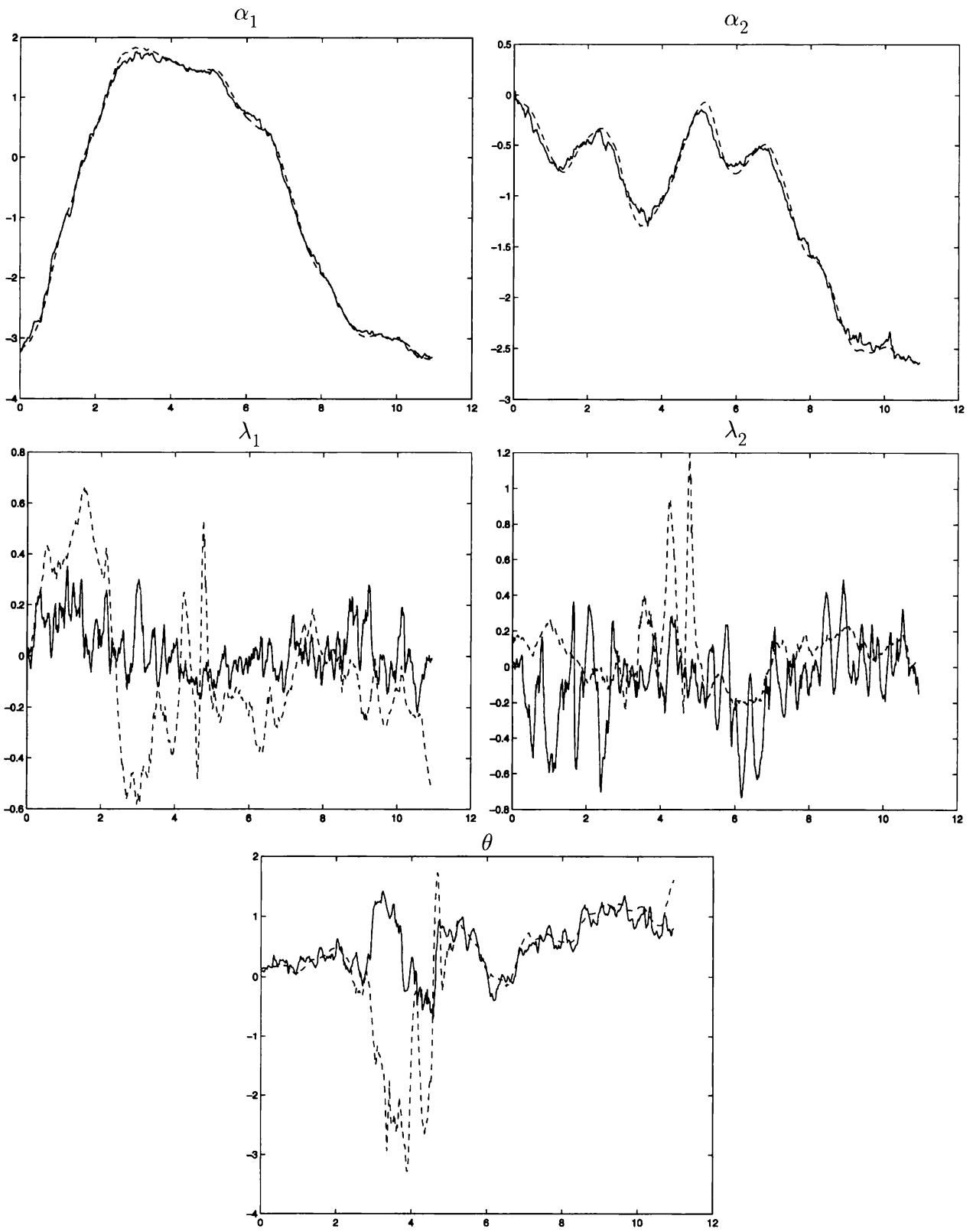

FIG. 6.3. Estimated generalized coordinates by the MCF (solid line) and the EKF (dotted line) as a function of time for the sequence in Clip One. From left to right, top to bottom: $\alpha_{1}, \alpha_{2}, \lambda_{1}, \lambda_{2}, \theta$.

located to the left of \#2 in panel C. The MCF was also distracted slightly by the other fish (see small wiggle to the left of \#2 in panel $\mathrm{C}$ ).

Clip Three (Figure 6.5). This sequence was the longest (1800 frames-approximately 30 seconds) and most complex. There were various sources of complexity: illumination 


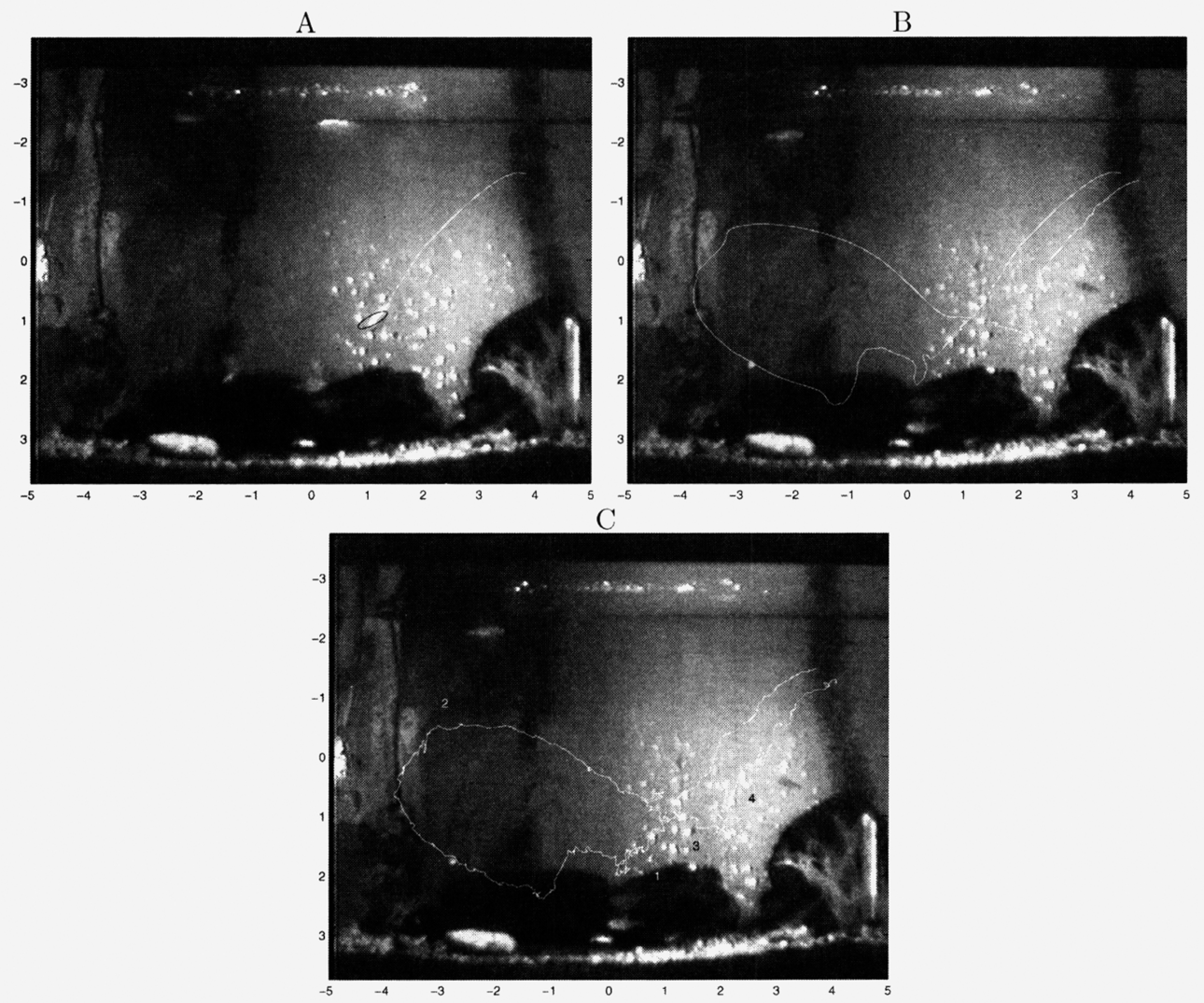

Fig. 6.4. Clip Two estimated trajectories. Panel A: Initial segment of the EKF estimated trajectory. Panel B: EKF estimated trajectory. Panel C: MCF estimated trajectory.

varied dramatically across the scene. The tracked fish was occasionally partly occluded, and at some point it became totally hidden behind a rock for a short period (about 30 frames); the scene contained other fish interacting closely with the fish being tracked (the other fish were of somewhat different shape than the tracked fish - their shapes were far from elliptical shapes. Also the tracked fish was brighter than the others, but this property was not built into our models).

Because of the complexity of the scene, and because no explicit model for multiple fish tracking was employed, one would not expect either the EKF or the MCF to be very successful (in some frames of the clip, it was difficult even for the human eye to single out the tracked fish from the other fish, even though the other fish were somewhat different). Indeed, the EKF eventually began to diverge, and crashed just about when the tracked fish was totally occluded. The MCF lost lock several times and jumped from fish to fish or to other background clutter, but each time it was able to recover and finish the track successfully.

Figure 6.5 shows six different frames of the MCF tracking. The tracked fish began in the upper right corner before turning and swimming diagonally downwards toward 

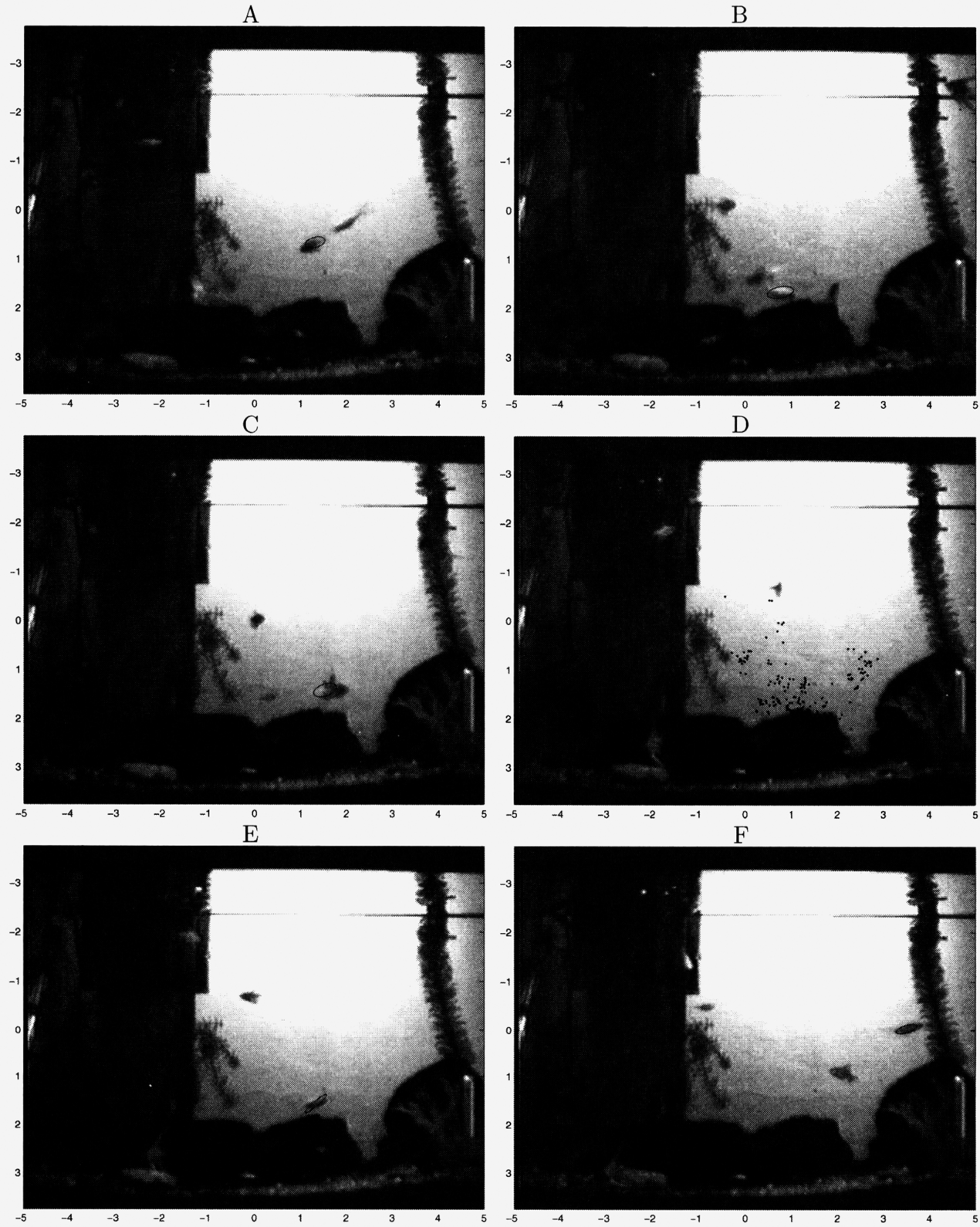

FIG. 6.5. Six frames of the MCF algorithm in the third clip

the center of the screen, following closely behind a small fish (see panel A). The fish was tracked accurately at the initial stages, but toward the center of the screen (where illumination changed dramatically), the MCF tracker jumped to the small fish in front of the tracked fish (see panel A-the tracked fish is brighter and behind the estimated ellipse locked at the small fish). When both fish reached below the center of the screen, the MCF switched back to the correct fish (panel B), probably because background noise 
cluttered the small fish (but also partially coincidentally). It proceeded to track the correct fish successfully for the most part thereafter, though at times it jumped to other fish (see panel C). The return to the correct fish was also helped by the fact that all other fish became occluded at some point. Successful tracking continued until the correct fish swam behind a rock and became totally occluded for approximately half a second (30 frames). At this point the MCF samples began to spread out and became quite diffuse (panel D; dark dots represent posterior samples). Just as the filter was beginning to converge to the angelfish at the lower left part of the frame (panel D), the correct fish re-emerged from behind the rock, and the MCF tracker quickly locked on it (panel E). It tracked it correctly to the end of the clip (panel F).

This experiment demonstrates the robustness of the MCF over the EKF. When the MCF loses the tracked object, it scarches for another object resembling the correct one; it has the ability to recover from a lost lock. But when tracking fails in the EKF, the algorithm typically diverges and the loss is permanent-it has very limited ability to recover from an incorrect lock.

6.2. Tracking Pool Table Balls. The second set of experiments concerns the tracking of moving balls on a pool table. We analysed several sequences of images, two of which are reported here. The sequences are less degraded than those in the fish experiments of the previous subsection, but the tracking of pool table balls presents a new difficulty to be described shortly. Some clutter was present in the form of cushions and pockets around the edge of the table; in addition, other balls besides the one being tracked were present in some of the clips (the other balls were originally stationary but were put in motion by collisions with the tracked ball; the tracked ball was first set in motion by striking it with a pool cue). Also, illumination varied considerably across the scene.

But the main difficulty in these experiments stemmed from the very abrupt changes in motion direction as the tracked ball bounced off cushions and/or the stationary balls: when the tracked ball is not bouncing off anything, it is subject to no external forces, but when it bounces off a cushion or another ball, it is subject to an external force of a very large magnitude. Consequently, the external force (dynamic noise) cannot be Gaussian. The main goal of these experiments was to test our handling of this problem within the EKF and MCF approaches.

In the EKF the dynamic noise is required to be Gaussian, and so we have no choice but to choose its variance judiciously. The difficulty was minimized by giving significant weight to the data model relative to the dynamics. To this end, we chose the dynamic variance to be equal to the data model variance. While this minimized the error of the Gaussian assumption (for the dynamic noise), it reduced the smoothness of the EKF estimated trajectories (as we pointed out in $\S 6.1$, if the variance of the dynamic noise is smaller than that of the data model, then the EKF estimated trajectories are smoother).

In the MCF the dynamic noise was taken to be a mixture of Gaussians. The components were chosen by the following rationale: no bouncing occurs with high probability (say $p$ ), and the external force (during no bouncing) is very small; so in this case the dynamic noise variance is chosen to be small. In contrast, bounces occur with small probability $1-p$, but induce large external force, and so, in this case, the dynamic noise 
variance is taken to be large. Accordingly, $W_{k}$ was chosen to be of the form

$$
W_{k}= \begin{cases}N\left(0, \sigma_{1} I\right) & \text { with probability } p \\ N\left(0, \sigma_{2} I\right) & \text { with probability } 1-p\end{cases}
$$

with $\sigma_{2} \gg \sigma_{1}$. In the experiments we chose $p=0.9, \sigma_{1}=1$, and $\sigma_{2}=20$.

The template was chosen to be a disc (naturally) of radius 0.2 (in units of image size $[-5,5] \times[-3.748,3.748])$. The transformations had three degrees of freedom - two for translation $\left(\alpha_{1}, \alpha_{2}\right)$, and one for scale $(\lambda)$, giving rise to a coordinate vector $q=$ $\left(\alpha_{1}, \alpha_{2}, \lambda\right)^{\top}$. The mass density was chosen $\rho(\xi)=1$, and the damping matrix $C(q)=$ $\frac{1}{2} M(q)$. The strain energy was given by $(3.10 \mathrm{~b})$ with $\epsilon(\lambda)=\lambda$ and $K_{0}=\kappa$. The stiffness constant $\kappa$ was chosen to be $\kappa=6$; this value was large enough to prevent the disc from shrinking to a point, but small enough so as not to inhibit tracking of scale changes.

Clip One (Figures 6.6-6.7). In this sequence there is only one ball on the table. The sequence contains 480 frames (approximately 8 seconds). The ball begins (see Fig. 6.6) at the bottom left of the frame, travelling at high speed; it bounces off cushions three times, and eventually rolls to a stop. Figure 6.6 shows the estimated trajectories for the EKF (white curve) and the MCF (black curve).

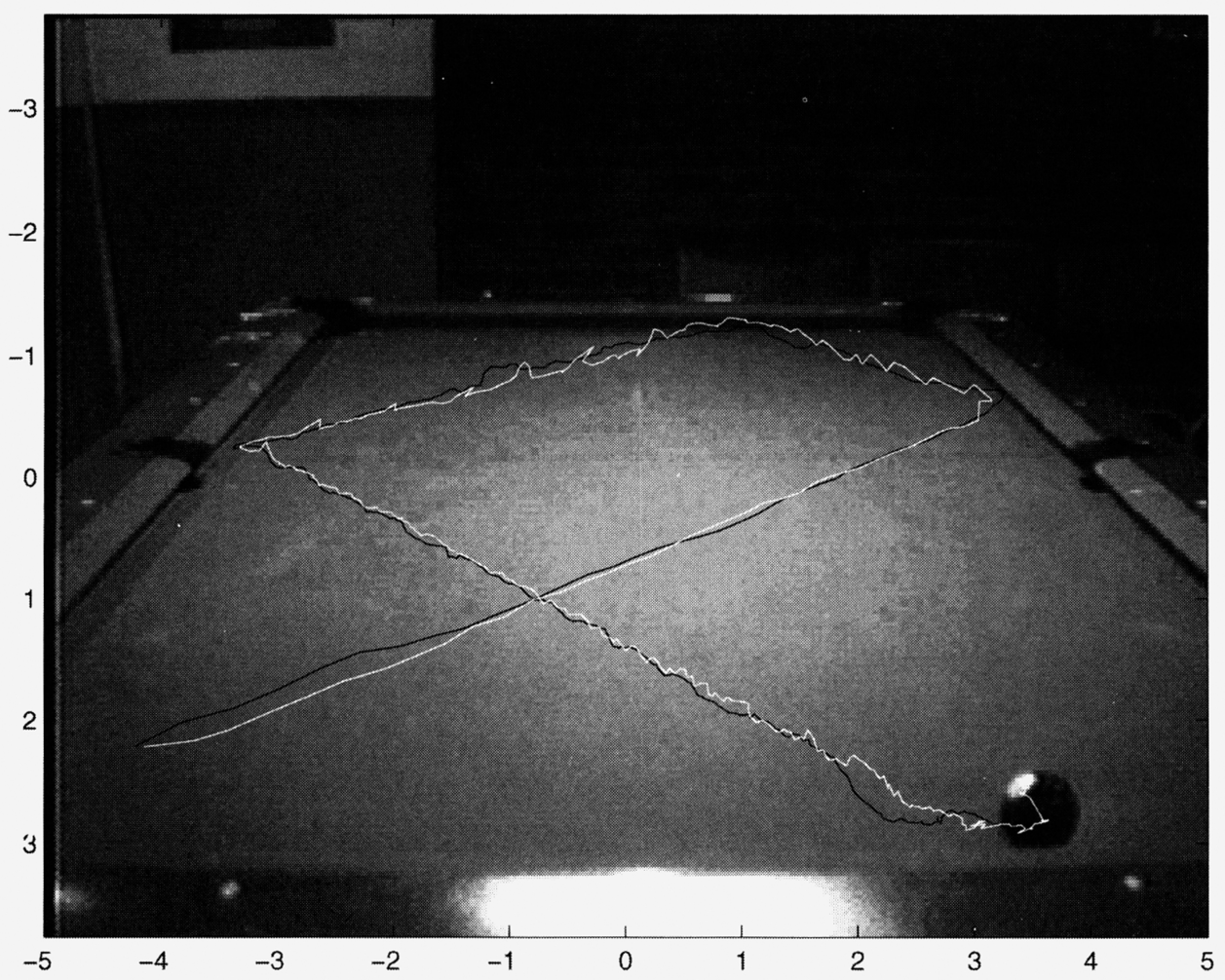

FIG. 6.6. Final frame in the sequence of Clip One, showing the estimated trajectories for the EKF (white curve) and the MCF (black curves). 
The MCF estimated trajectory is smoother than that of the EKF (the same behavior occurs in clip two below, as well as in other clips not reported here). This behavior is opposite to the one in the fish experiments (as §6.1), and, as mentioned above, the reason for this is the relative significant weight we gave to the data model by choosing (for EKF) the dynamic and data model variances equal; this choice implies that errors in the observation term have more effect on the tracker.

Figure 6.7 shows the estimates of the three coordinates $\alpha_{1}, \alpha_{2}$, and $\lambda$ for MCF (solid curve) and EKF.(dotted curve). The estimate of $\lambda$ is poorer than that of the location coordinates $\alpha_{1}$ and $\alpha_{2}$. This is due primarily to illumination effects. Any specularity on the upper part of the ball was frequently interpreted as part of the exterior rather than the interior of the ball (although the tracker would sometimes focus only on the specularity and disregard the rest of the ball). In order to improve the estimate of $\lambda$, one would need to go beyond a purely edge-based model used here and introduce an illumination model as well.
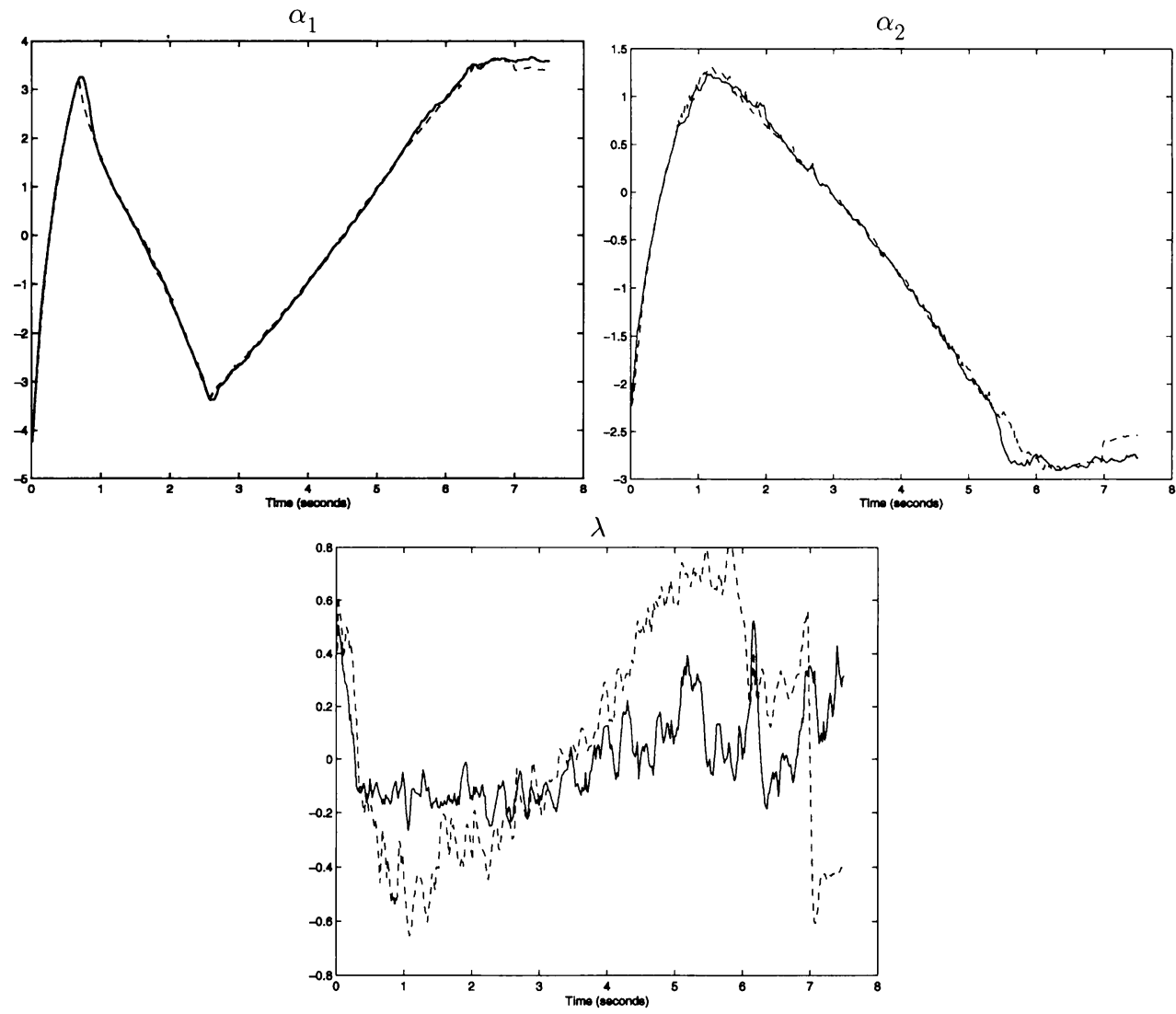

FIG. 6.7. Estimated generalized coordinates for the sequence in Clip One, by the MCF (solid curve) and the EKF (dotted curve). From left-to-right, top-to-bottom: $\alpha_{1}, \alpha_{2}, \lambda$.

Clip Two (Figures 6.8-6.9). In this sequence, in addition to the tracked ball, two other balls (originally stationary) were present. The tracked ball began (see Fig. 6.8) at the 
front right of the table and passed close to the two stationary balls, striking one of them. The tracked ball had slightly different color than the other two balls - it was lighter and thus had higher contrast edges.

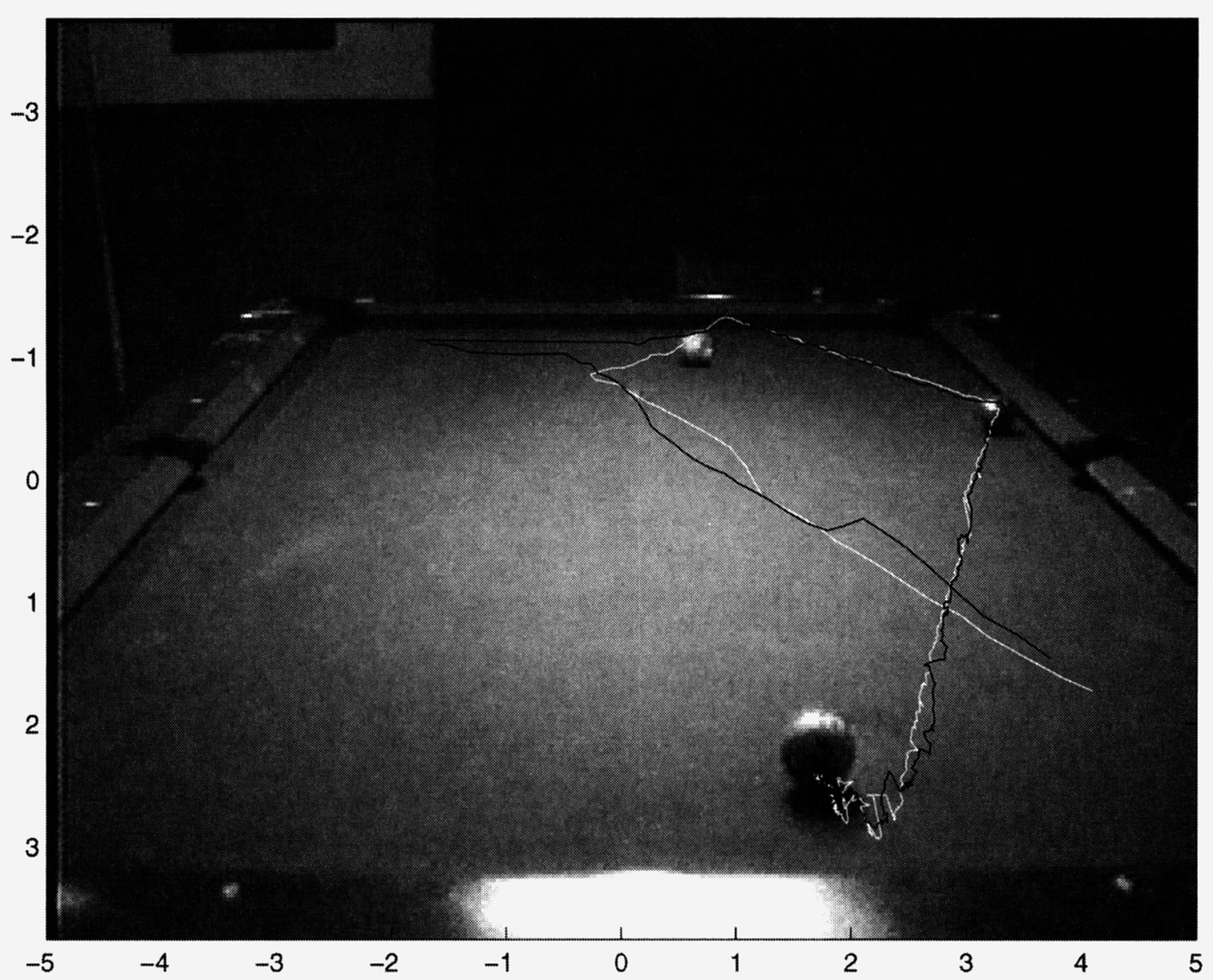

FIG. 6.8. Estimated trajectories for the sequence of Clip Two, by the EKF (white curve) and the MCF (black curve).

Figure 6.8 shows the estimated trajectories for the MCF (black curve) and the EKF (white curve). The one area where the MCF was less successful than the EKF was when the tracked ball struck one of the stationary balls. During this period there were three balls within the same region, and the MCF samples split into three clusters corresponding to the three balls (see panels in Fig. 6.9). For a while (see left-most part of the MCF trajectory in Fig. 6.8), the MCF tracked the wrong ball. However, it recovered from the incorrect lock (15 frames later), and resumed tracking successfully.

For this and other sequences (not reported here), both the MCF and the EKF performed ralatively well. The Gaussian mixture for the MCF dynamic noise was effective; it allowed successful tracking at bounces without sacrificing accuracy during normal motion. The EKF, while its assumption on the Gaussians of the noises did not allow for the bounce aspect of the motion to be modelled correctly, performed well on the strength of a robust and accurate data model. 

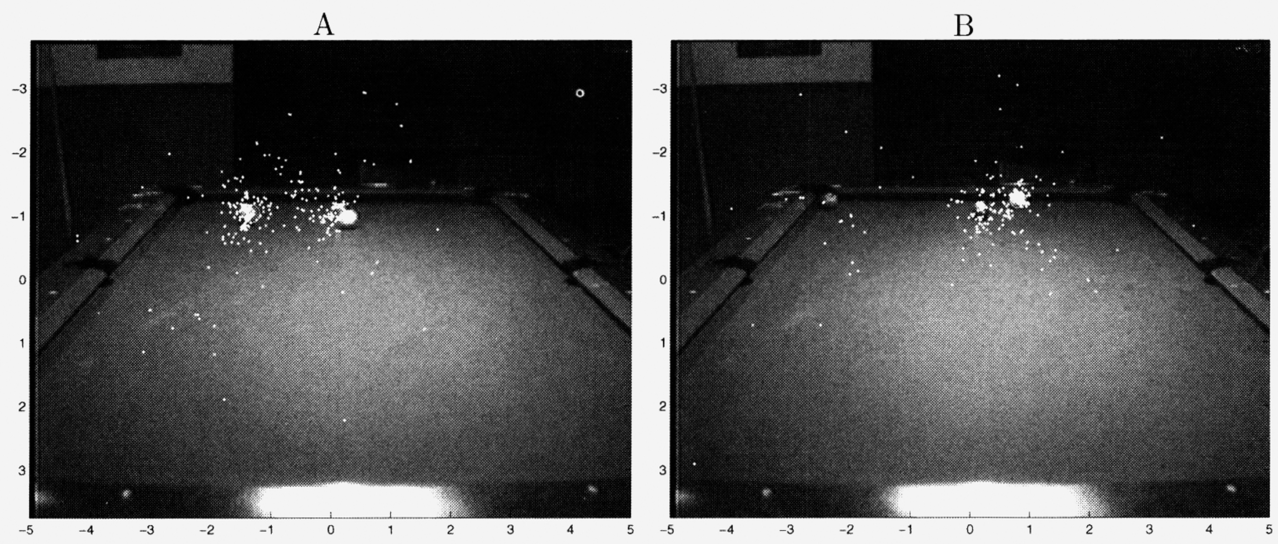

FIG. 6.9. Clustering of samples of the MCF during collisions with the other ball, in Clip Two.

Appendix. In this Appendix we complete our derivation of equations (3.3), (3.4), (3.7), and (3.11).

Equations (3.3) and (3.4). Taking the time derivative in (2.2b) and using (2.5b) and $(2.2 \mathrm{a})$, one easily derives

$$
\dot{x}=L(\xi ; \theta, \lambda) \dot{q} \quad, \quad \xi \in V
$$

where $V$ is the template and

$$
L=L(\xi ; \theta, \lambda)=(I, R(\theta) \sigma w(\xi), R(\theta) S(\xi))
$$

Here $I$ is the $2 \times 2$ identity matrix and $\sigma$ the Pauli matrix defined below (3.4). Then (3.2) yields (3.3) with

$$
\begin{aligned}
M & =\int_{V} \rho(\xi) L^{\top} L d \xi \\
& =\int_{V} \rho(\xi)\left(\begin{array}{ccc}
I & R \sigma w & R S \\
w^{\top} \sigma^{\top} R^{\top} & w^{\top} w & w^{\top} \sigma^{\top} S \\
S^{\top} R^{\top} & S^{\top} \sigma w & S^{\top} S
\end{array}\right) d \xi \\
& =\left(\begin{array}{ccc}
M_{\alpha, \alpha} & M_{\alpha, \theta} & M_{\alpha, \lambda} \\
M_{\alpha, \theta}^{\top} & M_{\theta, \theta} & M_{\theta, \lambda} \\
M_{\alpha, \lambda}^{\top} & M_{\theta, \lambda}^{\top} & M_{\lambda, \lambda}
\end{array}\right) .
\end{aligned}
$$


This quickly yields formulas (3.4) with $m, \mu, \mu_{i, j}$ defined below (3.4), and

$$
\begin{aligned}
\Sigma & =\int_{V} \rho(\xi) S(\xi) d \xi \\
\beta & =\int_{V} \rho(\xi) S^{\top}(\xi) \xi d \xi \\
\beta_{1} & =\int_{V} \rho(\xi) S^{\top}(\xi) \sigma \xi d \xi \\
\Gamma & =\int_{V} \rho(\xi) S^{\top}(\xi) S(\xi) d \xi \\
\Gamma_{1} & =\int_{V} \rho(\xi) S^{\top}(\xi) \sigma^{\top} S(\xi) d \xi
\end{aligned}
$$

Note that $\beta$ and $\beta_{1}$ are not independent; they involve the same moments. The same is true for $\Gamma$ and $\Gamma_{1}$. In the affine transformation case we have

$$
S(\xi)=\left(\begin{array}{ccc}
\xi_{1} & 0 & \xi_{2} \\
0 & \xi_{2} & \xi_{1}
\end{array}\right)
$$

and $\Sigma, \beta, \beta_{1}, \Gamma, \Gamma_{1}$ are given in terms of $\mu=\left(\mu_{1}, \mu_{2}\right)^{\top}$ and $\mu_{i, j}, i=1,2$ :

$$
\begin{aligned}
\Sigma & =\left(\begin{array}{ccc}
\mu_{1} & 0 & \mu_{2} \\
0 & \mu_{2} & \mu_{1}
\end{array}\right) \\
\beta & =\left(\begin{array}{c}
\mu_{11} \\
\mu_{22} \\
2 \mu_{12}
\end{array}\right), \beta_{1}=\left(\begin{array}{c}
-\mu_{12} \\
\mu_{12} \\
\mu_{11}-\mu_{22}
\end{array}\right) \\
\Gamma & =\left(\begin{array}{ccc}
\mu_{11} & 0 & \mu_{12} \\
0 & \mu_{22} & \mu_{12} \\
\mu_{12} & \mu_{12} & \mu_{11}+\mu_{22}
\end{array}\right), \Gamma_{1}=\left(\begin{array}{ccc}
0 & \mu_{12} & \mu_{11} \\
-\mu_{12} & 0 & -\mu_{22} \\
-\mu_{1}^{2} & \mu_{22} & 0
\end{array}\right) .
\end{aligned}
$$

Equation (3.7). Let $S_{\mu}(\xi), \mu=1,2$, denote the $\mu$-th row of $S(\xi)$, and view $S_{\mu}(\xi)$ as an $N_{\lambda} \times 1$ vector. Also set

$$
S_{\mu}^{(i)}=S_{\mu}^{(i)}(\xi)=\frac{\partial S_{\mu}(\xi)}{\partial \xi_{i}}, i=1,2 .
$$

Then a straightforward calculation yields

$$
\begin{aligned}
& \epsilon_{11}=S_{1}^{(1)} \cdot \lambda+\frac{1}{2} \sum_{\mu=1}^{2}\left(S_{\mu}^{(1)} \cdot \lambda\right)^{2} \\
& \epsilon_{22}=S_{2}^{(2)} \cdot \lambda+\frac{1}{2} \sum_{\mu=1}^{2}\left(S_{\mu}^{(2)} \cdot \lambda\right)^{2} \\
& \epsilon_{12}=\frac{1}{2}\left(S_{1}^{(2)}+S_{2}^{(1)}\right) \cdot \lambda+\frac{1}{2} \sum_{\mu=1}^{2}\left(S_{\mu}^{(1)} \cdot \lambda\right)\left(S_{\mu}^{(2)} \cdot \lambda\right)
\end{aligned}
$$

where $\cdot$ denotes inner product. These equations identify the matrices $D_{0}(\xi)$ and $Q_{0}(\xi)$ in (3.7). 
Equation (3.11). The $F(q, \dot{q})$ term is given by

$$
F(q, \dot{q})=\dot{M}(q) \dot{q}-\frac{1}{2} \frac{\partial}{\partial q}\left(\dot{q}^{\top} M(q) \dot{q}\right)
$$

or in terms of components

$$
(F(q, \dot{q}))_{j}=\sum_{i, k=1}^{3+N_{\lambda}}\left(\frac{\partial M_{j, i}}{\partial q_{k}}-\frac{1}{2} \frac{\partial M_{k, i}}{\partial q_{j}}\right) \dot{q}_{k} \dot{q}_{i}, j=1,2, \ldots, 3+N_{\lambda} .
$$

As we stated in $\S 3.2, F(q, \dot{q})$ depends on $\theta, \lambda, \dot{\theta}, \dot{\lambda}$ only, and is quadratic in $\dot{\theta}$ and $\dot{\lambda}$.

The last term in (3.11) is as follows: let $\tilde{e}(\lambda)$ and $\tilde{K}$ be as in (3.9b). Also let $\tilde{B}(\lambda)$ denote the Jacobian matrix of $\tilde{e}(\lambda)$, i.e.,

Then

$$
\tilde{B}(\lambda)=\frac{\partial \tilde{e}(\lambda)}{\partial \lambda}
$$

$$
\begin{aligned}
e(\lambda) & =\left(\begin{array}{c}
0 \\
0 \\
\tilde{e}(\lambda)
\end{array}\right) \\
K & =\left(\begin{array}{ccc}
0 & 0 & 0 \\
0 & 0 & 0 \\
0 & 0 & \tilde{K}
\end{array}\right) \\
B(\lambda) & =\left(\begin{array}{ccc}
0 & 0 & 0 \\
0 & 0 & 0 \\
0 & 0 & \tilde{B}(\lambda)
\end{array}\right) .
\end{aligned}
$$




\section{REFERENCES}

[1] Brian D. O. Anderson and John B. Moore. Optimal Filtering. Prentice-Hall, 1979.

[2] Andrew Blake, Rupert Curwen, and Andrew Zisserman. A framework for spatio-temporal control in the tracking of visual contours, Int. J. Computer Vision, 11(2):127-145, 1993.

[3] Andrew Blake and Michael Isard. Active Contours. Springer-Verlag, 1998.

[4] R. Bajcsy and D. Jones. Multiresolution Elastic Matching, Computer Vision, Graphics, and Image Processing, 48, 1-21, 1989.

[5] Andrew Blake, Michael Isard, and David Reynard. Learning to track the visual motion of contours, Artificial Intelligence, 78:101-133, 1995.

[6] Arthur P. Boresi and Ken P. Chong. Elasticity in Engineering Mechanics. Elsevier Science Publishing Co., 1987.

[7] James Carpenter, Peter Clifford, and Paul Fearnhead. An Improved Particle Filter for Non-Linear Problems, IEE Proceedings - Radar, Sonar, and Navigation, 146(1), 2-7, 1999.

[8] P. Del Moral. Measure-Valued Processes and Interacting Particle Systems: Application to Nonlinear Filtering Problem, The Annals of Applied Probability, 8, 2, 438-495, 1998.

[9] Robert J. Elliott, Lakhdar Aggoun, and John B. Moore. Hidden Markov Models: Estimation and Control. Springer-Verlag, 1995.

[10] Arthur Gelb, editor. Applied Optimal Estimation. The M.I.T. Press, 1974.

[11] Stuart Geman and Donald Geman. Stochastic Relaxation, Gibbs Distribution, and Bayesian Restoration of Images, IEEE Trans. PAMI, 6, 721-741, 1984.

[12] Jean Dickinson Gibbons and Subhabrata Chakraborti. Nonparametric Statistical Inference. Marcel Dekker, Inc., third edition, 1992.

[13] N. J. Gordon, D. J. Salmond, and A. F. M. Smith. Novel approach to nonlinear/non-Gaussian Bayesian state estimation, IEE Proceedings-F, 140(2):107-113, April 1993.

[14] Ulf Grenander. General Pattern Theory: A Study of Regular Structures. Oxford Press, 1993.

[15] Michael Isard. Visual Motion Analysis by Probabilistic Propagation of Conditional Density. Ph.D. Thesis 1998, Department of Engineering Science, Oxford University.

[16] Michael Isard and Andrew Blake. Contour tracking by stochastic propagation of conditional density, In Proc. European Conf. on Computer Vision, pages 343-356, 1996.

[17] Genshiro Kitagawa. Monte carlo filter and smoother for non-Gaussian nonlinear state space models, Journal of Computational and Graphical Statistics, 5(1):1-25, 1996.

[18] John MacCormick. Probabilistic Modeling and Stochastic Algorithms for Visual Localisation and Tracking, Ph.D. Thesis 2000, Department of Engineering Science, Oxford University.

[19] M. I. Miller, A. Srivastava, and U. Grenander. Conditional-expectation estimation via jumpdiffusion processes in multiple target tracking/recognition, IEEE Transactions on Signal Processing, 43(11):2678-2690, November 1995.

[20] Christopher Raphael and Stuart Geman. A grammatical approach to mine detection, SPIE Proceedings, Vol. 3079, 1997, pp. 316-332, Eds.: A. C. Dubey and R. L. Bamard.

[21] Christopher Robertson. Tracking of Objects from Image Sequences Using Lagrangean Dynamics and Nonlinear Filtering, Ph.D. Thesis 1998, Division of Applied Mathematics, Brown University.

[22] Reinhardt M. Rosenberg. Analytical Dynamics of Discrete Systems. Plenum Press, 1977.

[23] D. B. Rubin. Using the SIR Algorithm to Simulate Posterior Distributions, in Bayesian Statistics, 3, pp. 395-402, Oxford University Press, 1998, Eds.: J. M. Bernado, M. H. DeGroot, D. V. Lindley, and A. F. M. Smith.

[24] Ahmed A. Shabana. Dynamics of Multibody Systems. John Wiley \& Sons, 1989.

[25] Ahmed A. Shabana. Variation of Discrete and Continuous Systems. Springer-Verlag, 1997.

[26] Demetri Terzopoulos and Dimitri Metaxas. Dynamic 3D models with local and global deformations: deformable superquadrics, IEEE Transactions on Pattern Analysis and Machine Intelligence, 13(7):703-714, July 1991.

[27] Demetri Terzopoulos and Dimitri Metaxas. Tracking nonrigid 3D objects. In Andrew Blake and Alan Yuille, editors, Active Vision, chapter 5, pages 75-89. The M.I.T. Press, 1992.

[28] Demetri Terzopoulos and Richard Szeliski. Tracking with Kalman snakes. In Andrew Blake and Alan Yuille, editors, Active Vision, chapter 1, pages 3-20. The M.I.T. Press, 1992.

[29] Demetri Terzopoulos and Keith Waters. Analysis and synthesis of facial image sequences using physical and anatomical models, IEEE Transactions on Pattern Analysis and Machine Intelligence, 15(6):569-579, June 1993. 